\title{
Persistent homology and microlocal sheaf theory
}

\author{
Masaki Kashiwara and Pierre Schapira
}

September 10, 2018

\begin{abstract}
We interpret some results of persistent homology and barcodes (in any dimension) with the language of microlocal sheaf theory. For that purpose we study the derived category of sheaves on a real finite-dimensional vector space $\mathbb{V}$. By using the operation of convolution, we introduce a pseudo-distance on this category and prove in particular a stability result for direct images. Then we assume that $\mathbb{V}$ is endowed with a closed convex proper cone $\gamma$ with non empty interior and study $\gamma$-sheaves, that is, constructible sheaves with microsupport contained in the antipodal to the polar cone (equivalently, constructible sheaves for the $\gamma$-topology). We prove that such sheaves may be approximated (for the pseudo-distance) by "piecewise linear" $\gamma$-sheaves. Finally we show that these last sheaves are constant on stratifications by $\gamma$-locally closed sets, an analogue of barcodes in higher dimension.
\end{abstract}

\section{Contents}

1 Persistent homology $\quad 3$

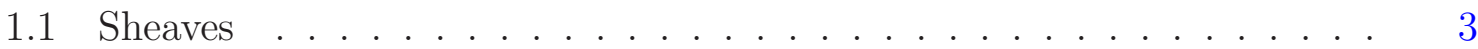

$1.2 \gamma$-topology . . . . . . . . . . . . . . . . . . 5

1.3 Persistent homology . . . . . . . . . . . . . . . . . . 7

1.4 Comparison with the Alexandrov topology . . . . . . . . . . . 8

1.5 The case of dimension one . . . . . . . . . . . . . . . . . 9

2 Sheaves on vector spaces $\quad 14$

2.1 Convolution . . . . . . . . . . . . . . . . . . . . 14

2.2 Distance and the stability theorem . . . . . . . . . . . . . . . . . . . . . . . . . . . . .

2.3 PL-sheaves . . . . . . . . . . . . . . . . . . . . . . . . . . . . . . . . . 19

2.4 The approximation theorem . . . . . . . . . . . . . . . . 19

2.5 Barcodes (multi-dimensional case) . . . . . . . . . . . . . . 20

Key words: microlocal sheaf theory, persistent homology, barcodes

MSC: 55N99, 18A99, 35A27

The research of M.K was supported by Grant-in-Aid for Scientific Research (B) 15H03608, Japan Society for the Promotion of Science.

The research of P.S was supported by the ANR-15-CE40-0007 "MICROLOCAL". 
3 Piecewise linear $\gamma$-sheaves 22

3.1 Complements on the $\gamma$-topology . . . . . . . . . . . . . . . . 22

3.2 Study of $\gamma$-sheaves . . . . . . . . . . . . . . . . . . . . . . . 25

3.3 Piecewise linear $\gamma$-sheaves . . . . . . . . . . . . . . . . . 27

\section{Introduction}

Persistent homology and barcodes are recent concrete applications of algebraic topology. The aim of this paper is to show that many results of this theory are easily interpreted in the language of microlocal sheaf theory and that, in this formulation, one may extend the theory to higher dimension.

Although the theory is quite new, there is already a vast literature on persistent homology. See in particular the survey papers [EH08, Ghr08, Oud15].

We understand persistent homology as follows. One has a finite subset $S$ of an Euclidian space $X$ and one wants to understand its structure. For that purpose, one replaces each point $x \in S$ with a closed ball of center $x$ and radius $t$ and makes $t$ going to infinity. The union of these balls gives a closed set $Z \subset X \times \mathbb{R}$, and one wants to understand how the homology of the union of the balls varies with $t$, which is equivalent to understanding the direct image of the constant sheaf $\mathbf{k}_{Z}$ associated with $Z$ by the projection $X \times \mathbb{R} \rightarrow \mathbb{R}$. From this point of view, we are not far from Morse theory for sheaves (see [GM88, KS90]). Moreover, the sheaf one obtains has particular properties. It is constructible and is associated with a topology whose open sets are the intervals ]$-\infty, a[$ with $a \in[-\infty,+\infty]$. As we shall see, the category of such sheaves is equivalent to a category (that we shall define) of barcodes. Note that the idea of using sheaf theory in this domain is not new and already appeared in the thesis of Justin Curry [Cur13].

As described above, persistent homology takes its values on $\mathbb{R}$ and barcodes are defined on the ordered space $(\mathbb{R}, \leq)$. However, the necessity of treating more than one parameter $t$ naturally appears (see for e.g. [Les15, LW15]).

A higher dimensional generalization of the ordered space $(\mathbb{R}, \leq)$ is the data of a finite-dimensional real vector space $\mathbb{V}$ and a closed convex proper cone $\gamma \subset \mathbb{V}$ with non-empty interior. We call here a $\gamma$-sheaf an object of the derived category of sheaves on $\mathbb{V}$ whose microsupport is contained in $\gamma^{\circ a}$, the antipodal to the polar cone to $\gamma$. As we shall see, this category is equivalent to the derived category of sheaves on $\mathbb{V}_{\gamma}$, the space $\mathbb{V}$ endowed with the so-called $\gamma$-topology.

The main goal of this paper is to describe constructible $\gamma$-sheaves on $\mathbb{V}$. Constructible sheaves on real analytic manifolds are now well-understood (the story began on complex manifolds with [Kas75]) but, as we shall see, $\gamma$-sheaves have a very specific behavior and are not so easy to treat. We shall mainly restrict ourselves to piecewise linear sheaves (PL-sheaves for short), those which are locally constant on a stratification associated with a locally finite family of hyperplanes.

The main results of this paper may be described as follows.

(a) In $\S 1$ we first recall and complete some results of [KS90, $\S 3.5]$ on the $\gamma$-topology, showing that the category of $\gamma$-sheaves is equivalent to the derived category of 
sheaves on $\mathbb{V}_{\gamma}$. Then we recall, in our language, some basic results of persistent homology (that is, essentially Morse theory for sheaves) and construct the category of barcodes on $\mathbb{R}_{\gamma}$, for $\gamma=\mathbb{R}_{\leq 0}$. By proving a variant of a result of CrawleyBoevey [CB14, Th. 1.1], using a result of Guillermou [Gui16, Cor. 7.3] based on Gabriel's theorem on representations of quivers, we prove that our category of barcodes is equivalent to that of constructible $\gamma$-sheaves.

(b) In $\S 2$, we define a kind of pseudo-distance dist on sheaves on $\mathbb{V}$, after having endowed $\mathbb{V}$ with a norm $\|\cdot\|$. The main tool for this construction is the convolution of sheaves and the main difference between our distance and the other classical ones, is that our distance is constructed in the derived setting. For example, two sheaves concentrated in different degrees may have a finite distance. We prove a stability result for direct images, namely, given two continuous maps $f_{1}, f_{2}: X \rightarrow \mathbb{V}$ and a sheaf $F$ on $X$, then $\operatorname{dist}\left(\mathrm{R} f_{1 *} F, \mathrm{R} f_{2 *} F\right) \leq\left\|f_{1}-f_{2}\right\|$.

Next, we introduce the notion of PL-sheaves and prove that any constructible sheaf may be approximated by a PL-sheaf as well as a similar result for PL- $\gamma$-sheaves (now, $\gamma$ is polyhedral).

Finally, we propose a notion of barcodes in higher dimension and construct the category of such barecodes. Unfortunately, although this category is naturally embedded into that of PL-sheaves, we show on examples that this embedding is not an equivalence.

(c) In $\S 3$, we first study with some details the $\gamma$-topology on $\mathbb{V}$ and constructible $\gamma$ sheaves. For example, we show that if $F$ is such a sheaf, then for any $x \in \mathbb{V}, F$ is locally constant on $x+\gamma^{a}$ in a neighborhood of $x$.

The main result of this section is that given a PL- $\gamma$-sheaf, there exists a stratification of $\mathbb{V}$ by $\gamma$-locally closed polytopes on which the sheaf is constant.

\section{Acknowledgments}

The second named author warmly thanks Gregory Ginot for having organized a seminar on persistent homology, at the origin of this paper, and Benoît Jubin for fruitful discussions on this subject. In this seminar, Nicolas Berkouk and Steve Oudot pointed out the problem of approximating constructible sheaves with objects which would be similar to higher dimensional barcodes, what we do, in some sense, here. Moreover, the links between $\gamma$-sheaves and persistent modules (Proposition 1.15) were clarified during discussions with Nicolas Berkouk.

\section{Persistent homology}

\subsection{Sheaves}

The aim of this subsection is simply to fix a few notations.

- Throughout the paper, $\mathbf{k}$ denotes a field. We denote by $\operatorname{Mod}(\mathbf{k})$ the abelian category of $\mathbf{k}$-vector spaces. 
- For an abelian category $\mathscr{C}$, we denote by $\mathrm{D}^{\mathrm{b}}(\mathscr{C})$ its bounded derived category. However, we write $\mathrm{D}^{\mathrm{b}}(\mathbf{k})$ instead of $\mathrm{D}^{\mathrm{b}}(\operatorname{Mod}(\mathbf{k}))$.

- If $\pi: E \rightarrow M$ is a vector bundle over $M$, we identify $M$ with the zero-section of $E$ and we set $\dot{E}:=E \backslash M$. We denote by $\dot{\pi}: \dot{E} \rightarrow M$ the restriction of $\pi$ to $\dot{E}$.

- For a vector bundle $E \rightarrow M$, we denote by $a: E \rightarrow E$ the antipodal map, $a(x, y)=(x,-y)$. For a subset $Z \subset E$, we simply denote by $Z^{a}$ its image by the antipodal map. In particular, for a cone $\gamma$ in $E$, we denote by $\gamma^{a}=-\gamma$ the opposite cone. For such a cone, we denote by $\gamma^{\circ}$ the polar cone (or dual cone) in the dual vector bundle $E^{*}$ :

$$
\gamma^{\circ}=\left\{(x ; \xi) \in E^{*} ;\langle\xi, v\rangle \geq 0 \text { for all } v \in \gamma_{x}\right\} .
$$

- Let $M$ be a real manifold $M$ of dimension $\operatorname{dim} M$. We shall use freely the classical notions of microlocal sheaf theory, referring to [KS90]. We denote by $\operatorname{Mod}\left(\mathbf{k}_{M}\right)$ the abelian category of sheaves of $\mathbf{k}$-modules on $M$ and by $\mathrm{D}^{\mathrm{b}}\left(\mathbf{k}_{M}\right)$ its bounded derived category. For short, an object of $\mathrm{D}^{\mathrm{b}}\left(\mathbf{k}_{M}\right)$ is called a "sheaf" on $M$.

- For a locally closed subset $Z \subset M$, one denotes by $\mathbf{k}_{Z}$ the constant sheaf with stalk $\mathbf{k}$ on $Z$ extended by 0 on $M \backslash Z$. One defines similarly the sheaf $L_{Z}$ for $L \in \mathrm{D}^{\mathrm{b}}(\mathbf{k})$.

- We denote by or $_{M}$ the orientation sheaf on $M$ and by $\omega_{M}$ the dualizing complex on $M$. Recall that $\omega_{M} \simeq \operatorname{or}_{M}[\operatorname{dim} M]$. One shall use the duality functors

$$
\mathrm{D}_{M}^{\prime}(\bullet)=\mathrm{R} \mathscr{H} o m\left(\bullet, \mathbf{k}_{M}\right), \quad \mathrm{D}_{M}(\bullet)=\mathrm{R} \mathscr{H} o m\left(\cdot, \omega_{M}\right) .
$$

- For $F \in \mathrm{D}^{\mathrm{b}}\left(\mathbf{k}_{M}\right)$ we denote by $\mu \operatorname{supp}(F)^{3}$ its microsupport, a closed conic coisotropic subset of $T^{*} M$.

- For $F \in \mathrm{D}^{\mathrm{b}}\left(\mathbf{k}_{M}\right)$, one denotes by $\operatorname{Sing}(F)$ the singular locus of $F$, that is, the complement of the largest open subset on which $F$ is locally constant.

\section{Constructible sheaves}

We refer the reader to [KS90] for terminologies not explained here.

Definition 1.1. Let $M$ be a real analytic manifold and let $F \in \operatorname{Mod}\left(\mathbf{k}_{M}\right)$. One says that $F$ is weakly $\mathbb{R}$-constructible if there exists a subanalytic stratification $M=\bigsqcup_{\alpha} M_{\alpha}$ such that for each stratum $M_{\alpha}$, the restriction $\left.F\right|_{M_{\alpha}}$ is locally constant. If moreover, the stalk $F_{x}$ is of finite rank for all $x \in M$, then one says that $F$ is $\mathbb{R}$-constructible.

Notation 1.2. (i) One denotes by $\operatorname{Mod}_{\mathbb{R c}}\left(\mathbf{k}_{M}\right)$ the abelian category of $\mathbb{R}$-constructible sheaves and by $\operatorname{Mod}_{\mathbb{R c}, \mathrm{c}}\left(\mathbf{k}_{M}\right)$ the full subcategory of $\operatorname{Mod}_{\mathbb{R c}}\left(\mathbf{k}_{M}\right)$ consisting of sheaves with compact support. Both are thick abelian subcategories of $\operatorname{Mod}\left(\mathbf{k}_{M}\right)$.

\footnotetext{
${ }^{3} \mu \operatorname{supp}(F)$ was denoted by $\mathrm{SS}(F)$ in $[\mathrm{KS} 90]$.
} 
(ii) One denotes by $\mathrm{D}_{\mathbb{R} c}^{\mathrm{b}}\left(\mathbf{k}_{M}\right)$ the full triangulated subcategory of $\mathrm{D}^{\mathrm{b}}\left(\mathbf{k}_{M}\right)$ consisting of sheaves with $\mathbb{R}$-constructible cohomology and by $\mathrm{D}_{\mathbb{R} c, \mathrm{c}}^{\mathrm{b}}\left(\mathbf{k}_{M}\right)$ the full triangulated subcategory of $\mathrm{D}_{\mathbb{R c}}^{\mathrm{b}}\left(\mathbf{k}_{M}\right)$ consisting of sheaves with compact support.

A theorem of [Kas84] (see also [KS90, Th. 8.4.5]) asserts that the natural functor $\mathrm{D}^{\mathrm{b}}\left(\operatorname{Mod}_{\mathbb{R c}}\left(\mathbf{k}_{M}\right)\right) \rightarrow \mathrm{D}_{\mathbb{R c}}^{\mathrm{b}}\left(\mathbf{k}_{M}\right)$ is an equivalence of categories.

When $F \in \mathrm{D}_{\mathbb{R} \mathbf{c}}^{\mathrm{b}}\left(\mathbf{k}_{M}\right), \mu \operatorname{supp}(F)$ and $\operatorname{Sing}(F)$ are subanalytic. The first result is proved in loc. cit. and the second one follows from $\operatorname{Sing}(F)=\dot{\pi}\left(\mu \operatorname{supp}(F) \cap \dot{T}^{*} M\right)$. (Recall that $\dot{\pi}$ is the projection $\dot{T}^{*} M:=T^{*} M \backslash M \rightarrow M$.)

\section{$1.2 \gamma$-topology}

The so-called $\gamma$-topology has been studied with some details in [KS90, § 3.4].

Let $\mathbb{V}$ be a finite-dimensional real vector space. We denote by $s$ the addition map.

$$
s: \mathbb{V} \times \mathbb{V} \rightarrow \mathbb{V}, \quad(x, y) \mapsto x+y,
$$

and by $a: \mathbb{V} \rightarrow \mathbb{V}$ the antipodal map $x \mapsto-x$.

Hence, for two subsets $A, B$ of $\mathbb{V}$, one has $A+B=s(A \times B)$. A subset $A$ of $V$ is called a cone if $0 \in A$ and $\mathbb{R}_{>0} A \subset A$. A convex cone $A$ is proper if $A \cap A^{a}=\{0\}$.

Throughout the paper, we consider a cone $\gamma \subset \mathbb{V}$ and we assume:

(1.3) $\gamma$ is closed proper convex with non-empty interior.

Sometimes we shall make the extra assumption that $\gamma$ is polyhedral, meaning that it is a finite intersection of closed half-spaces.

We say that a subset $A$ of $\mathbb{V}$ is $\gamma$-invariant if $A+\gamma=A$. Note that a subset $A$ is $\gamma$-invariant if and only if $\mathbb{V} \backslash A$ is $\gamma^{a}$-invariant.

The family of $\gamma$-invariant open subsets of $\mathbb{V}$ defines a topology, which is called the $\gamma$-topology ${ }^{4}$ on $\mathbb{V}$. One denotes by $\mathbb{V}_{\gamma}$ the space $\mathbb{V}$ endowed with the $\gamma$-topology and one denotes by

$$
\varphi_{\gamma}: \mathbb{V} \rightarrow \mathbb{V}_{\gamma}
$$

the continuous map associated with the identity. Note that the closed sets for this topology are the $\gamma^{a}$-invariant closed subsets of $\mathbb{V}$.

Definition 1.3. Let $A$ be a subset of $\mathbb{V}$.

(a) One says that $A$ is $\gamma$-open (resp. $\gamma$-closed), if $A$ is open (resp. closed) for the $\gamma$-topology.

(b) One says that $A$ is $\gamma$-locally closed if $A$ is the intersection of a $\gamma$-open subset and a $\gamma$-closed subset.

(c) One says that $A$ is $\gamma$-flat if $A=(A+\gamma) \cap\left(A+\gamma^{a}\right)$.

\footnotetext{
${ }^{4}$ See Subsection 1.4 for related constructions.
} 
(d) One says that a closed set $A$ is $\gamma$-proper if the map $s$ is proper on $A \times \gamma^{a}$.

Remark 1.4. (i) A closed subset $A$ is $\gamma$-proper if and only if $A \cap(x+\gamma)$ is compact for any $x \in \mathbb{V}$.

(ii) Let $A$ be a subset of $\mathbb{V}$ and assume that $\bar{A}$ is $\gamma$-proper. Then $\overline{A+\gamma^{a}}=\bar{A}+\gamma^{a}$.

(iii) If $A$ is closed and if there exist a closed convex proper cone $\gamma_{1}$ with $\gamma \subset \operatorname{Int}\left(\gamma_{1}\right) \cup\{0\}$ and $x \in \mathbb{V}$ such that $A \cap\left(x+\gamma_{1}\right)=\varnothing$, then $A$ is $\gamma$-proper.

(iv) One has $\operatorname{Int}(\gamma)=\operatorname{Int}(\gamma)+\gamma$ and $\overline{\operatorname{Int}(\gamma)}=\gamma$.

We shall use the notations:

$$
\left\{\begin{array}{l}
\mathrm{D}_{\gamma^{\circ a}}^{\mathrm{b}}\left(\mathbf{k}_{\mathbb{V}}\right):=\left\{F \in \mathrm{D}^{\mathrm{b}}\left(\mathbf{k}_{\mathbb{V}}\right) ; \mu \operatorname{supp}(F) \subset \mathbb{V} \times \gamma^{\circ a}\right\}, \\
\mathrm{D}_{\mathbb{R c}, \gamma^{\circ \mathrm{a}}}^{\mathrm{b}}\left(\mathbf{k}_{\mathbb{V}}\right):=\mathrm{D}_{\mathbb{R}_{\mathrm{c}}}^{\mathrm{b}}\left(\mathbf{k}_{\mathbb{V}}\right) \cap \mathrm{D}_{\gamma^{\circ a}}^{\mathrm{b}}\left(\mathbf{k}_{\mathbb{V}}\right), \\
\operatorname{Mod}_{\gamma^{\circ \mathrm{a}}}\left(\mathbf{k}_{\mathbb{V}}\right):=\operatorname{Mod}\left(\mathbf{k}_{\mathbb{V}}\right) \cap \mathrm{D}_{\gamma^{\circ a}}^{\mathrm{b}}\left(\mathbf{k}_{\mathbb{V}}\right), \\
\operatorname{Mod}_{\mathbb{R c}, \gamma^{\circ \mathrm{a}}}\left(\mathbf{k}_{\mathbb{V}}\right):=\operatorname{Mod}_{\mathbb{R} \mathrm{c}}\left(\mathbf{k}_{\mathbb{V}}\right) \cap \operatorname{Mod}_{\gamma^{\circ \mathrm{a}}}\left(\mathbf{k}_{\mathbb{V}}\right) .
\end{array}\right.
$$

We call an object of $\mathrm{D}_{\gamma^{\circ} \mathrm{a}}^{\mathrm{b}}\left(\mathbf{k}_{\mathbb{V}}\right)$ a $\gamma$-sheaf.

It follows from [KS90, Prop. 5.4.14] that for $F, G \in \mathrm{D}_{\gamma^{\circ a}}^{\mathrm{b}}\left(\mathbf{k}_{\mathbb{V}}\right)$ and $H \in \mathrm{D}_{\gamma^{\circ}}^{\mathrm{b}}\left(\mathbf{k}_{\mathbb{V}}\right)$, the sheaves $F \otimes G$ and R $\mathscr{H} o m(H, F)$ belong to $\mathrm{D}_{\gamma^{\circ} \mathrm{b}}^{\mathrm{b}}\left(\mathbf{k}_{\mathbb{V}}\right)$.

The next result is implicitly proved in [KS90], without assuming that $\operatorname{Int}(\gamma)$ is non empty.

Theorem 1.5. Let $\gamma$ be a closed convex proper cone in $\mathbb{V}$. The functor $\mathrm{R} \varphi_{\gamma_{*}}: \mathrm{D}_{\gamma^{\circ a}}^{\mathrm{b}}\left(\mathbf{k}_{\mathbb{V}}\right) \rightarrow$ $\mathrm{D}^{\mathrm{b}}\left(\mathbf{k}_{\mathbb{V}_{\gamma}}\right)$ is an equivalence of triangulated categories with quasi-inverse $\varphi_{\gamma}^{-1}$.

Proof. (i) The proof of [KS90, Prop. 5.2.3] shows that $\varphi_{\gamma}^{-1}: \mathrm{D}^{\mathrm{b}}\left(\mathbf{k}_{\mathbb{V}_{\gamma}}\right) \rightarrow \mathrm{D}_{\gamma^{\circ a}}^{\mathrm{b}}\left(\mathbf{k}_{\mathbb{V}}\right)$ is well defined. The same statement asserts that for $F \in \mathrm{D}_{\gamma^{\circ a}}^{\mathrm{b}}\left(\mathbf{k}_{\mathbb{V}}\right)$, one has $\varphi_{\gamma}^{-1} \mathrm{R} \varphi_{\gamma_{*}} F \stackrel{\sim}{\longrightarrow} F$. (ii) By [KS90, Prop. 3.5.3 (iii)], for $F \in \mathrm{D}^{\mathrm{b}}\left(\mathbf{k}_{\mathbb{V}_{\gamma}}\right)$, one has $F \stackrel{\sim}{\longrightarrow} \varphi_{\gamma_{*}} \varphi_{\gamma}^{-1} F$.

Corollary 1.6. The functor $\varphi_{\gamma_{*}}: \operatorname{Mod}_{\gamma^{\circ a}}\left(\mathbf{k}_{\mathbb{V}}\right) \rightarrow \operatorname{Mod}\left(\mathbf{k}_{\mathbb{V}_{\gamma}}\right)$ is an equivalence of abelian categories with quasi-inverse $\varphi_{\gamma}^{-1}$.

Proof. (i) By Theorem 1.5, the functor $\varphi_{\gamma}^{-1}: \operatorname{Mod}\left(\mathbf{k}_{\mathbb{V}_{\gamma}}\right) \rightarrow \operatorname{Mod}\left(\mathbf{k}_{\mathbb{V}}\right)$ takes its values in $\operatorname{Mod}_{\gamma^{\circ a}}\left(\mathbf{k}_{\mathbb{V}}\right)$. Moreover, the same statement asserts that for $F \in \operatorname{Mod}\left(\mathbf{k}_{\mathbb{V}_{\gamma}}\right), F \stackrel{\sim}{\longrightarrow}$ $\mathrm{R} \varphi_{\gamma_{*}} \varphi_{\gamma}^{-1} F$. Therefore, $F \stackrel{\sim}{\longrightarrow} \varphi_{\gamma_{*}} \varphi_{\gamma}^{-1} F$.

(ii) By taking the 0-th cohomology of the isomorphism $\varphi_{\gamma}^{-1} \mathrm{R} \varphi_{\gamma_{*}} F \stackrel{\sim}{\longrightarrow} F$ and using the fact that $\varphi_{\gamma}^{-1}$ commutes with $H^{0}$, we get the isomorphism $\varphi_{\gamma}^{-1} \varphi_{\gamma_{*}} F \stackrel{\sim}{\longrightarrow}$.

Corollary 1.7. The equivalence of categories in Theorem 1.5 preserves the natural $t$ structures of both categories. In particular, for $F \in \mathrm{D}^{\mathrm{b}}\left(\mathbf{k}_{\mathbb{V}}\right)$, the condition $F \in \mathrm{D}_{\gamma^{\circ a}}^{\mathrm{b}}\left(\mathbf{k}_{\mathbb{V}}\right)$ is equivalent to the condition: $\mu \operatorname{supp}\left(H^{j}(F)\right) \subset \gamma^{\circ a}$ for any $j \in \mathbb{Z}$.

Proof. This follows from Corollary 1.6.

Corollary 1.8. Let $A$ be a $\gamma$-locally closed subset of $\mathbb{V}$. Then $\mu \operatorname{supp}\left(\mathbf{k}_{A}\right) \subset \mathbb{V} \times \gamma^{\circ a}$.

Proof. The subset $A$ is locally closed in $V_{\gamma}$. Let us denote by $\mathbf{k}_{A, \gamma} \in \operatorname{Mod}\left(\mathbf{k}_{V_{\gamma}}\right)$ the constant sheaf supported on $A$. Then we have $\mathbf{k}_{A} \simeq \varphi_{\gamma}^{-1}\left(\mathbf{k}_{A, \gamma}\right)$. 
Remark 1.9. Thanks to Theorem 1.5, the reader may ignore microlocal sheaf theory, at least in a first reading. Indeed, if this theory plays a central role in the proofs of the statements, it does not appear in the statements, after replacing $\mathrm{D}_{\gamma^{\circ a}}^{\mathrm{b}}\left(\mathbf{k}_{\mathbb{V}}\right)$ with $\mathrm{D}^{\mathrm{b}}\left(\mathbf{k}_{\mathbb{V}_{\gamma}}\right)$.

\subsection{Persistent homology}

Let $\mathbb{V}$ be a real finite-dimensional vector space and let $\gamma$ be a cone satisfying hypothesis (1.3). We also assume that $\gamma$ is subanalytic ${ }^{5}$.

Let $M$ be a real analytic manifold that and let $f: M \rightarrow \mathbb{V}$ be a continuous subanalytic map. We denote by $\Gamma_{f}^{+} \subset M \times \mathbb{V}$ the $\gamma$-epigraph of $f$.

$$
\begin{aligned}
\Gamma_{f}^{+} & =\{(x, y) \in M \times \mathbb{V} ; f(x)-y \in \gamma\} \\
& =\Gamma_{f}+\gamma^{a} .
\end{aligned}
$$

We denote by $p: M \times \mathbb{V} \rightarrow \mathbb{V}$ the projection.

Lemma 1.10. One has $\mu \operatorname{supp}\left(\mathbf{k}_{\Gamma_{f}^{+}}\right) \subset T^{*} M \times\left(\mathbb{V} \times \gamma^{\circ a}\right)$.

Proof. The set $\Gamma_{f}^{+}$being $\gamma$-closed, the result follows from Corollary 1.8 .

Theorem 1.11. Let $M$ be a real analytic manifold and let $f: M \rightarrow \mathbb{V}$ be a continuous subanalytic map. Assume that

$$
\text { for each } K \subset \mathbb{V} \text { compact, the set }\{x \in M ; f(x) \in K+\gamma\} \text { is compact. }
$$

Then $\mathrm{R}_{*} \mathrm{k}_{\Gamma_{f}^{+}}$belongs to $\mathrm{D}_{\mathbb{R} c, \gamma^{\circ a}}^{\mathrm{b}}\left(\mathbf{k}_{\mathbb{V}}\right)$.

Proof. Let $K \subset \mathbb{V}$ be a compact subset. Then

$$
\begin{aligned}
\left\{(x, y) \in \Gamma_{f}^{+} ; y \in K\right\} & =\{(x, y) \in M \times \mathbb{V} ; f(x) \in y+\gamma, y \in K\} \\
\subset & \{x \in M ; f(x) \in K+\gamma\} \times K
\end{aligned}
$$

Hence, the map $p$ is proper on $\Gamma_{f}^{+}$.

Applying [KS90, Prop. 5.4.4], we get that $\mathrm{R} p_{*} \mathbf{k}_{\Gamma_{f}^{+}}$belongs to $\mathrm{D}_{\gamma^{\circ a}}^{\mathrm{b}}\left(\mathbf{k}_{\mathbb{V}}\right)$. Moreover, this object is $\mathbb{R}$-constructible by loc. cit. Prop. 8.4.8.

Example 1.12. Let $M$ and $f$ be as above with $\mathbb{V}=\mathbb{R}$ and $\gamma=\{t \leq 0\}$. In this case, $\Gamma_{f}^{+}=\{(x, t) \in M \times \mathbb{R} ; f(x) \leq t\}$ is the epigraph of $f$. Hypothesis (1.6) is translated as:

(1.7) for each $t \in \mathbb{R}$, the set $\{x \in M ; f(x) \leq t\}$ is compact.

Set $K=\{x \in M ; f(x) \leq 0\}$. By the hypothesis, $K$ is compact. Let $a=\inf _{x \in K} f(x)$. Then $f(x) \geq a$ for all $x \in M$.

\footnotetext{
${ }^{5}$ In practice the cone $\gamma$ will be polyhedral.
} 
A more explicit example may be obtained as follows. Assume that $M$ is endowed with a subanalytic distance and let $S$ be a finite subset of $M$. Then one can choose $f(x)=d(x, S)$ in which case,

$$
\Gamma_{f}^{+}=\{(x, t) \in M \times \mathbb{R} ; d(x, S) \leq t\}=\bigcup_{s \in S} B(s ; t)
$$

where $B(s ; t)$ is the closed ball of center $s$ and radius $t$. One can also endow each $s \in S$ with some weight $\rho(s) \in \mathbb{R}_{\geq 0}$ and replace $B(s ; t)$ with $B(s ; \rho(s) t)$.

Example 1.13. Let $\mathbb{V}=\mathbb{R}^{n}, \gamma=\left(\mathbb{R}_{\leq 0}\right)^{n}$ and let $f=\left(f_{1}, \cdots, f_{n}\right)$, each $f_{i}$ being continuous and subanalytic and satisfying hypothesis (1.7). Then $f$ satisfies the hypothesis of Theorem 1.11. Moreover, in this case, $\operatorname{supp}\left(\mathrm{R} p_{*} \mathbf{k}_{\Gamma_{f}^{+}}\right) \subset y+\gamma^{a}$ for some $y \in \mathbb{V}$.

Remark 1.14. We have assumed that $M$ is real analytic and $f: M \rightarrow \mathbb{V}$ is continuous and subanalytic in order that $\mathrm{R} p_{*} \mathbf{k}_{\Gamma_{f}^{+}}$be constructible. If $\mathbb{V}=\mathbb{R}$, these hypotheses may be weakened by simply assuming that the critical values of $f$ are discrete.

Also note that, although different from the class of $C^{\infty}$-maps, the class of continuous subanalytic maps is very large.

\subsection{Comparison with the Alexandrov topology}

Most of the authors define (higher dimensional) persistent homology modules as functors from the order set $\left(\mathbb{R}^{n}, \leq\right.$ ) (where $\leq$ is the product order corresponding to the cone $\gamma=\left(\mathbb{R}_{\leq 0}\right)^{n}$ ) to the category of vector spaces over the field $\mathbf{k}$. Some authors also consider the Alexandrov topology on $\mathbb{R}^{n}$ associated with $\gamma$. Let us clarify the link with these different approaches.

Set $\mathbb{V}, \gamma$ and $\mathbb{V}_{\gamma}$ be as above. Denote by $\mathbb{W}_{\gamma}$ the set $\mathbb{V}$ endowed with the Alexandrov topology associated with the cone $\gamma$. Hence, $A \subset \mathbb{V}$ is open in $\mathbb{W}_{\gamma}$ if and only if $A=A+\gamma$. On the other hand denote by $X_{\gamma}$ the set $\mathbb{V}$ endowed with the order $x \leq y$ if and only if $x+\gamma \subset y+\gamma$. We look at $X_{\gamma}$ as a category, hence as a presite, hence as a site for the trivial Grothendieck topology. Therefore, a sheaf on $X_{\gamma}$ is nothing but a presheaf on $X_{\gamma}$, that is, a functor $X_{\gamma}^{\text {op }} \rightarrow \operatorname{Mod}(\mathbf{k})$ and, of course, $X_{\gamma}^{\text {op }}$ is equivalent to $\left(\mathbb{V}, \leq^{\mathrm{op}}\right)$.

We have morphisms of sites

$$
\begin{aligned}
& \alpha: \mathbb{V}_{\gamma} \rightarrow \mathbb{W}_{\gamma}, \quad \mathrm{Op}_{\mathbb{W}_{\gamma}} \ni A+\gamma \mapsto A+\operatorname{Int}(\gamma) \in \mathrm{Op}_{\mathbb{V}_{\gamma}}, \\
& \beta: \mathbb{W}_{\gamma} \rightarrow X_{\gamma}, \quad X_{\gamma} \ni x \mapsto x+\gamma, \\
& \rho: \mathbb{V}_{\gamma} \rightarrow X_{\gamma} \quad X_{\gamma} \ni x \mapsto x+\operatorname{Int}(\gamma) .
\end{aligned}
$$

Note that $\rho \simeq \beta \circ \alpha$. The morphisms $\alpha, \beta$ and $\rho$ induce functors

$$
\operatorname{Mod}\left(\mathbb{V}_{\gamma}\right) \underset{\alpha^{-1}}{\stackrel{\alpha_{*}}{\rightleftarrows}} \operatorname{Mod}\left(\mathbb{W}_{\gamma}\right), \operatorname{Mod}\left(\mathbb{W}_{\gamma}\right) \underset{\beta^{-1}}{\stackrel{\beta_{*}}{\rightleftarrows}} \operatorname{Mod}\left(X_{\gamma}\right), \operatorname{Mod}\left(\mathbb{V}_{\gamma}\right) \underset{\rho^{-1}}{\stackrel{\rho_{*}}{\rightleftarrows}} \operatorname{Mod}\left(X_{\gamma}\right)
$$


As already mentioned, the category of persistent modules is the category of presheaves (equivalently, of sheaves) on $X_{\gamma}$. Consider the diagram of categories

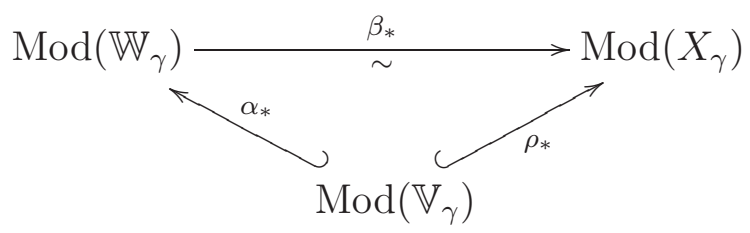

Proposition 1.15. ${ }^{6}$

(a) The functor $\rho_{*}$ is fully faithful and is not an equivalence.

(b) The functor $\beta_{*}$ is an equivalence. ${ }^{7}$

(c) The functor $\alpha_{*}$ is fully faithful.

Proof. In the sequel, for $x \in \mathbb{V}$ we set $U_{x}=x+\operatorname{Int}(\gamma)$.

(a)-(i) Denote by $\rho^{\dagger}$ the inverse image by $\rho$ in the category of presheaves. Let $F \in$ $\operatorname{Mod}\left(\mathbf{k}_{\mathbb{V}_{\gamma}}\right)$ and let $x \in \mathbb{V}$. One has $\left(\rho^{\dagger} \rho_{*} F\right)\left(U_{x}\right) \simeq F\left(U_{x}\right)$. Since the family $\left\{U_{x}\right\}_{x \in \mathbb{V}}$ is a basis of the topology of $\mathbb{V}_{\gamma}$, we conclude that $\rho^{-1} \rho_{*} F \simeq F$.

(a)-(ii) Let $x \in \mathbb{V}$ and consider the skyscraper presheaf on $X_{\gamma}$ at $x$, denoted here $\delta_{x}$. Then $\rho^{-1} \delta_{x}(x) \simeq 0$ since for a sheaf $F$ on $\mathbb{V}_{\gamma}, F\left(U_{x}\right) \simeq \lim _{\longleftarrow} F\left(U_{y}\right)$ where the limit ranges over the family $y \in U_{x}$.

(b)-(i) Denote by $\beta^{\dagger}$ the inverse image by $\beta$ in the category of presheaves. Let $F \in$ $\operatorname{Mod}\left(\mathbf{k}_{\mathbb{W}_{\gamma}}\right)$ and let $x \in \mathbb{V}$. One has $\left(\beta^{\dagger} \beta_{*} F\right)(x+\gamma) \simeq F(x+\gamma)$. Since the family $\{x+\gamma\}_{x \in \mathbb{V}}$ is a basis of the topology of $\mathbb{W}_{\gamma}$, we conclude that $\beta^{-1} \beta_{*} F \simeq F$.

(b)-(ii) Let $G \in \operatorname{Mod}\left(\mathbf{k}_{X_{\gamma}}\right)$. Then $\left(\beta_{*} \beta^{-1} G\right)(x) \simeq\left(\beta^{-1} G\right)(x+\gamma) \simeq G(x)$.

(c) follows from (a) and (b) since $\rho_{*} \simeq \beta_{*} \circ \alpha_{*}$.

It follows from Proposition 1.15 that one may consider the category of $\gamma$-sheaves as a full subcategory of that of persistent modules.

\subsection{The case of dimension one}

The aim of this paper is to describe the category $\mathrm{D}_{\mathbb{R} c, \gamma^{\text {oa }}}^{\mathrm{b}}\left(\mathbf{k}_{\mathbb{V}}\right)$. We first treat the case of the dimension one, where things are particularly simple. We shall study the category of $\gamma$-sheaves and construct a category of barcodes in dimension one, proving the equivalence of these categories in Theorem 1.25. Note that various constructions of categories of barcodes already exist in the literature. See in particular [BS14, BdSS15, BL16].

We denote by $t$ a coordinate on $\mathbb{R}$ and by $(t ; \tau)$ the associated homogeneous coordinates on $T^{*} \mathbb{R}$. Therefore, $F \in \operatorname{Mod}_{\mathbb{R c}}\left(\mathbf{k}_{\mathbb{R}}\right)$ if there exists a discrete set $Z \subset \mathbb{R}$ such that $F$ is locally constant on $\mathbb{R} \backslash Z$ and moreover, the stalk of $F$ at each point of $\mathbb{R}$ is finite-dimensional.

In the sequel, an interval means a non-empty convex subset of $\mathbb{R}$.

\footnotetext{
${ }^{6}$ As already mentioned, these results were clarified during discussions of the second named author with Nicolas Berkouk.

${ }^{7}$ This statement is due to [Cur13, Th.4.2.10].
} 
Proposition 1.16. Let $F \in \operatorname{Mod}_{\mathbb{R}}\left(\mathbf{k}_{\mathbb{R}}\right)$ and assume that $F$ has compact support. Then, there exist a finite set $A$ and a family of intervals $\left\{I_{\alpha}\right\}_{\alpha \in A}$ such that $F \simeq \bigoplus_{\alpha \in A} \mathbf{k}_{I_{\alpha}}$. Moreover such a decomposition is unique.

This result is due to Guillermou [Gui16, Cor. 7.3] who deduced it from Gabriel's theorem on the representation of quivers. We shall extend it to the non compact case, obtaining a result very similar to a theorem of Crawley-Boevey [CB14, Theorem 1.1].

Theorem 1.17. Let $F \in \operatorname{Mod}_{\mathbb{R c}}\left(\mathbf{k}_{\mathbb{R}}\right)$. Then, there exists a locally finite family of intervals $\left\{I_{\alpha}\right\}_{\alpha \in A}$ such that $F \simeq \bigoplus_{\alpha \in A} \mathbf{k}_{I_{\alpha}}$. Moreover such a decomposition is unique.

Proof. For $n \in \mathbb{Z}_{>0}$, set $\left.U_{n}=\right]-n, n\left[\right.$ and $F_{n}=F \otimes \mathbf{k}_{U_{n}}$. Then by the theorem above, there exists a finite family $\left\{I_{\alpha}^{(n)}\right\}_{\alpha \in A_{n}}$ of intervals in $U_{n}$ such that $F_{n} \simeq \oplus_{\alpha \in A_{n}} \mathbf{k}_{I_{\alpha}^{(n)}}$. Then there exists an injective map $A_{n} \longmapsto A_{n+1}$ (hereafter we identify $A_{n}$ as a subset of $A_{n+1}$ by this injective map) such that $I_{\alpha}^{(n)}=I_{\alpha}^{(n+1)} \cap U_{n}$ for $\alpha \in A_{n}$. Set $A=\bigcup_{n \in \mathbb{Z}_{>0}} A_{n}$, Then, for any $\alpha \in A$, there exists a unique interval $I_{\alpha}$ such that $I_{\alpha} \cap U_{n}=I_{\alpha}^{(n)}$ for any $n$ such that $\alpha \in A_{n}$. Then $\left\{I_{\alpha}\right\}_{\alpha \in A}$ is a locally finite family of intervals. Note that $A_{n}=\left\{\alpha \in A ; I_{\alpha} \cap U_{n} \neq \varnothing\right\}$ and $I_{\alpha}^{(n)}=I_{\alpha} \cap U_{n}$ for $\alpha \in A_{n}$.

Let us show that $F$ is isomorphic to $\bigoplus_{\alpha \in A} \mathbf{k}_{I_{\alpha}}$. For any $n$, there exists an isomorphism

$$
\varphi_{n}:\left.\left.\left.F\right|_{U_{n}} \stackrel{\sim}{\longrightarrow} \bigoplus_{\alpha \in A} \mathbf{k}_{I_{\alpha}}\right|_{U_{n}} \simeq \bigoplus_{\alpha \in A_{n}} \mathbf{k}_{I_{\alpha}}\right|_{U_{n}}
$$

The restriction map

$$
\operatorname{Hom}_{\operatorname{Mod}\left(\mathbf{k}_{U_{m}}\right)}\left(\left.\mathbf{k}_{I_{\alpha}}\right|_{U_{m}},\left.\mathbf{k}_{I_{\beta}}\right|_{U_{m}}\right) \rightarrow \operatorname{Hom}_{\operatorname{Mod}\left(\mathbf{k}_{U_{n}}\right)}\left(\left.\mathbf{k}_{I_{\alpha}}\right|_{U_{n}},\left.\mathbf{k}_{I_{\beta}}\right|_{U_{n}}\right)
$$

is injective for $m \geq n$ and $\alpha, \beta \in A_{n}$. Indeed, the injectivity follows from the commutative diagram

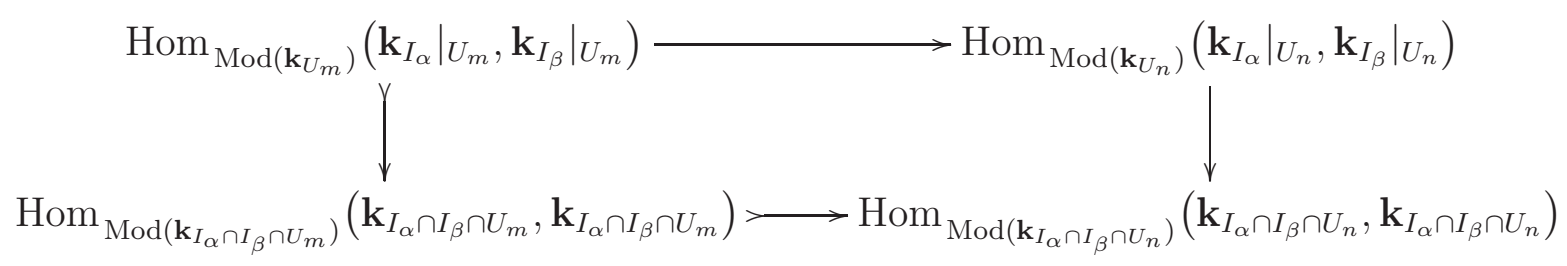

Here the injectivity of the bottom arrows follows from the fact that $I_{\alpha} \cap I_{\beta} \cap U_{m}$ is empty if $I_{\alpha} \cap I_{\beta} \cap U_{n}$ is empty.

Hence the restriction map

$$
\operatorname{End}_{\operatorname{Mod}\left(\mathbf{k}_{U_{m}}\right)}\left(\left.\bigoplus_{\alpha \in A_{n}} \mathbf{k}_{I_{\alpha}}\right|_{U_{m}}\right) \rightarrow \operatorname{End}_{\operatorname{Mod}\left(\mathbf{k}_{U_{n}}\right)}\left(\left.\bigoplus_{\alpha \in A_{n}} \mathbf{k}_{I_{\alpha}}\right|_{U_{n}}\right)
$$

is injective for $m \geq n$. Therefore, if an automorphism $f$ of $\left.\left.\bigoplus_{\alpha \in A} \mathbf{k}_{I_{\alpha}}\right|_{U_{n}} \simeq \bigoplus_{\alpha \in A_{n}} \mathbf{k}_{I_{\alpha}}\right|_{U_{n}}$, as well as $f^{-1}$, lifts to an endomorphism of $\left.\bigoplus_{\alpha \in A_{n}} \mathbf{k}_{I_{\alpha}}\right|_{U_{m}}$, then it lifts to an automorphism of $\left.\bigoplus_{\alpha \in A_{n}} \mathbf{k}_{I_{\alpha}}\right|_{U_{m}}$. 
By the injectivity of (1.8) and the fact that $\operatorname{dim}\left(\operatorname{End}_{\operatorname{Mod}\left(\mathbf{k}_{U_{n}}\right)}\left(\left.\bigoplus_{\alpha \in A_{n}} \mathbf{k}_{I_{\alpha}}\right|_{U_{n}}\right)\right)<\infty$, there exists $m \geq n$ such that

$$
\operatorname{End}_{\operatorname{Mod}\left(\mathbf{k}_{U_{k}}\right)}\left(\left.\bigoplus_{\alpha \in A_{n}} \mathbf{k}_{I_{\alpha}}\right|_{U_{k}}\right) \rightarrow \operatorname{End}_{\operatorname{Mod}\left(\mathbf{k}_{U_{m}}\right)}\left(\left.\bigoplus_{\alpha \in A_{n}} \mathbf{k}_{I_{\alpha}}\right|_{U_{m}}\right)
$$

is an isomorphism for any $k \geq m$.

Thus we conclude that the image $K_{k, n}$ of the restriction map

$$
\operatorname{Aut}\left(\left.\bigoplus_{\alpha \in A} \mathbf{k}_{I_{\alpha}}\right|_{U_{k}}\right) \rightarrow \operatorname{Aut}\left(\left.\bigoplus_{\alpha \in A} \mathbf{k}_{I_{\alpha}}\right|_{U_{n}}\right)
$$

is equal to $K_{m, n}$ for any $k \geq m$. Let $P_{n}$ be the set of isomorphisms $\left.F\right|_{U_{n}} \stackrel{\sim}{\longrightarrow}$ $\left.\bigoplus_{\alpha \in A} \mathbf{k}_{I_{\alpha}}\right|_{U_{n}}$. Then $\left\{P_{n}\right\}_{n \in \mathbb{Z}_{>0}}$ is a projective system of non-empty sets. Moreover, for any $n$, there exists $m \geq n$ such that $\operatorname{Im}\left(P_{k} \rightarrow P_{n}\right)=\operatorname{Im}\left(P_{m} \rightarrow P_{n}\right)$ for any $k \geq m$. Set $\tilde{P}_{n}=\operatorname{Im}\left(P_{m} \rightarrow P_{n}\right) \subset P_{n}$. Then $\left\{\tilde{P}_{n}\right\}_{n \in \mathbb{Z}>0}$ is a projective system of non-empty sets such that the map $\tilde{P}_{m} \rightarrow \tilde{P}_{n}$ is surjective for any $m \geq n$.

Hence, by replacing $\varphi_{n}$, we can choose $\varphi_{n} \in \tilde{P}_{n}$ inductively so that we have $\left.\varphi_{n+1}\right|_{U_{n}}=\varphi_{n}$ for every $n$. Thus we conclude that $F \simeq \bigoplus_{\alpha \in A} \mathbf{k}_{I_{\alpha}}$.

Corollary 1.18. Let $F, G \in \operatorname{Mod}_{\mathbb{R c}}\left(\mathbf{k}_{\mathbb{R}}\right)$. Then $\operatorname{Ext}^{j}(F, G)=0$ for $j>1$.

Proof. By Theorem 1.17, we may assume that $F=\bigoplus_{a \in A} F_{a}$ and $G=\prod_{b \in B} G_{b}$ with $F_{a}=\mathbf{k}_{I_{a}}$ and $G=\mathbf{k}_{J_{b}}$ where $I_{a}$ and $J_{b}$ are intervals. Since

$$
\operatorname{Ext}^{j}\left(\bigoplus_{a \in A} F_{a}, \prod_{b \in B} G_{b}\right) \simeq \prod_{(a, b) \in A \times B} \operatorname{Ext}^{j}\left(F_{a}, G_{b}\right)
$$

we are reduced to prove the result with $F$ and $G$ replaced with $F_{a}$ and $G_{b}$ and in this case the result is obvious.

Example 1.19. In Corollary 1.18, one cannot replace $j>1$ with $j \geq 1$. Indeed, one has $\operatorname{Ext}^{1}\left(\mathbf{k}_{[0,+\infty[}, \mathbf{k}_{]-\infty, 0[}\right) \simeq \mathbf{k}$. (See Lemma 3.13 for a generalization in higher dimension.)

Corollary 1.18 classically implies

Corollary 1.20. Let $F \in \mathrm{D}_{\mathbb{R c}}^{\mathrm{b}}\left(\mathbf{k}_{\mathbb{R}}\right)$. Then $F \simeq \bigoplus_{j} H^{j}(F)[-j]$.

In other words, any object of the bounded derived category is isomorphic to the direct sum of its shifted cohomology objects. The same phenomena appears with the bounded derived category of the category of $\mathbb{Z}$-modules or that of $\mathbf{k}[X]$-modules, for $\mathbf{k}$ a field.

\section{Barcodes}

We denote by $\mathbb{R}_{\gamma}$ the set $\mathbb{R}$ endowed with the $\gamma$-topology where $\left.\left.\gamma=\right]-\infty, 0\right]$. Hence, the $\gamma$-open sets are the open subsets $\left.\mathbb{R}_{<t}:=\right]-\infty, t[$ and the $\gamma$-closed sets are the closed subsets $\mathbb{R}_{\geq t}:=[t,+\infty[$, with $t \in \overline{\mathbb{R}}$. The non-empty $\gamma$-locally closed sets are the intervals $[a, b[$ with $-\infty \leq a<b \leq+\infty$. 
Definition 1.21. A $\gamma$-barcode $(A, I)$, or simply a barcode, is the data of a set of indices $A$ and a family $I=\left\{I_{\alpha}\right\}_{\alpha \in A}$ of intervals $I_{\alpha}=\left[a_{\alpha}, b_{\alpha}\left[\subset \mathbb{R}\right.\right.$ with $-\infty \leq a_{\alpha}<b_{\alpha} \leq+\infty$, these data satisfying

the family $\left\{I_{\alpha}\right\}_{\alpha \in A}$ is locally finite on $\mathbb{R}$, that is, for any compact subset $K$ of $\mathbb{R},\left\{\alpha \in A ; I_{\alpha} \cap K \neq \varnothing\right\}$ is finite.

In particular, $A$ is countable. The support of the barcode $(A, I)$, denoted by $\operatorname{supp}(A, I)$, is the closed set $\bigcup_{\alpha \in A} \overline{I_{\alpha}}$.

Note that we do not ask $I_{\alpha} \neq I_{\beta}$ for $\alpha \neq \beta$. Otherwise, we should have to endow each $I_{\alpha}$ with a multiplicity $m_{\alpha} \in \mathbb{N}$.

We shall now construct a category of $\gamma$-barcodes, with Theorem 1.25 in view.

Let us say that a barcode $(A, I)$ is elementary if $A \simeq$ pt. We shall identify the elementary barcode (pt, $I$ ) with the interval $I$.

Given two elementary barcodes $[a, b[$ and $[c, d[$, we set

$$
\operatorname{Hom}_{\mathbf{B a r}_{\gamma}}\left(\left[a, b\left[,\left[c, d[)= \begin{cases}\mathbf{k} & \text { if } a \leq c<b \leq d \\ 0 & \text { otherwise. }\end{cases}\right.\right.\right.\right.
$$

Note that

$$
\operatorname{Hom}_{\mathbf{B a r}_{\gamma}}\left(\left[a, b\left[,\left[c, d[) \simeq \operatorname{Hom}\left(\mathbf{k}_{[a, b[}, \mathbf{k}_{[c, d[}\right) .\right.\right.\right.\right.
$$

Given two barcodes $(A, I)$ and $(B, J)$ with $I_{\alpha}=\left[a_{\alpha}, b_{\alpha}\left[\right.\right.$ and $J_{\beta}=\left[c_{\beta}, d_{\beta}[\right.$, we set

$$
\operatorname{Hom}_{\mathbf{B a r}_{\gamma}}((A, I),(B, J))=\prod_{(\alpha, \beta) \in A \times B} \operatorname{Hom}_{\mathbf{B a r}_{\gamma}}\left(\left[a_{\alpha}, b_{\alpha}\left[,\left[c_{\beta}, d_{\beta}[)\right.\right.\right.\right.
$$

For $u \in \operatorname{Hom}_{\mathbf{B a r}_{\gamma}}((A, I),(B, J))$ and $v \in \operatorname{Hom}_{\mathbf{B a r}_{\gamma}}((B, J),(C, K))$, the composition $v \circ u$ is defined as follows. Let $u=\left\{c_{\alpha, \beta}\right\}_{(\alpha, \beta) \in A \times B}$ and $v=\left\{c_{\beta, \gamma}\right\}_{(\beta, \gamma) \in B \times C}$ with $c_{\alpha, \beta}, c_{\beta, \gamma} \in \mathbf{k}$. One sets $v \circ u=\left\{c_{\alpha, \gamma}\right\}_{(\alpha, \gamma) \in A \times C}$ with

$$
c_{\alpha, \gamma}=\sum_{\beta \in B} c_{\alpha, \beta} \cdot c_{\beta, \gamma} \quad \text { if } \operatorname{Hom}_{\mathbf{B a r}_{\gamma}}\left(I_{\alpha}, K_{\gamma}\right)=\mathbf{k}
$$

For a given $(\alpha, \gamma)$, the sum in (1.13) is finite. Indeed, given $[a, b[$ and $[e, f[$, consider the intervals $\left[c_{\beta}, d_{\beta}\right.$ [ with $-\infty \leq a \leq c_{\beta}<b \leq d_{\beta} \leq+\infty$ and $-\infty \leq c_{\beta} \leq e<d_{\beta} \leq f \leq$ $+\infty$. If $b<+\infty$ or $-\infty<e$ then either $b$ or $e$ belongs to $\left[c_{\beta}, d_{\beta}\right.$ [ and the set of such $\beta$ is finite thanks to (1.9). Now assume that $b=+\infty$ and $-\infty=e$. Then $c_{\beta}=-\infty$ and $d_{\beta}=+\infty$ and again the family of such $\beta$ must be finite.

Notation 1.22. We denote by $\mathrm{Bar}_{\gamma}$ the category constructed above and call it the category of $\gamma$-barcodes.

Remark 1.23. The category $\mathbf{B a r}_{\gamma}$ contains much more morphisms than the category constructed in [BL16]. 
Lemma 1.24. The category $\mathbf{B a r}_{\gamma}$ is additive.

Proof. (i) The 0-barcode is $0=(A, I)$ with $A=\varnothing$.

(ii) Given two barcodes $(A, I)$ and $(B, J)$, we set $(A, I) \oplus(B, J)=(A \sqcup B, I \sqcup J)$.

Then we define a functor $\Psi: \operatorname{Bar}_{\gamma} \rightarrow \operatorname{Mod}_{\mathbb{R} c, \gamma^{\circ a}}\left(\mathbf{k}_{\mathbb{R}}\right)$ as follows. We set

$$
\Psi(A, I)=\bigoplus_{\alpha \in A} \mathbf{k}_{I_{\alpha}}
$$

By (1.11), both $\operatorname{Hom}_{\mathbf{B a r}_{\gamma}}\left(\left[a, b\left[,\left[c, d[)\right.\right.\right.\right.$ and $\operatorname{Hom}\left(\mathbf{k}_{[a, b[}, \mathbf{k}_{[c, d[}\right)$ are simultaneously $\mathbf{k}$ or 0. Hence, we define $\Psi: \operatorname{Hom}_{\mathbf{B a r}_{\gamma}}\left(\left[a, b\left[,\left[c, d[) \rightarrow \operatorname{Hom}\left(\mathbf{k}_{[a, b[}, \mathbf{k}_{[c, d[}\right)\right.\right.\right.\right.$ as the identity of $\mathbf{k}$.

Note that $\Psi$ commutes with the composition of morphisms.

Then we extend $\Psi$ by linearity:

$$
\begin{aligned}
\Psi: \operatorname{Hom}_{\mathbf{B a r}_{\gamma}}((A, I),(B, J)) & =\prod_{\alpha \in A, \beta \in B} \operatorname{Hom}_{\mathbf{B a r}_{\gamma}}\left(I_{\alpha}, J_{\beta}\right) \\
& \simeq \prod_{\alpha \in A, \beta \in B} \operatorname{Hom}\left(\mathbf{k}_{I_{\alpha}} \mathbf{k}_{J_{\beta}}\right) \\
& \simeq \operatorname{Hom}\left(\bigoplus_{\alpha \in A} \mathbf{k}_{I_{\alpha}}, \bigoplus_{\beta \in B} \mathbf{k}_{J_{\beta}}\right) .
\end{aligned}
$$

Here, we have used the fact that the sum is locally finite.

This construction shows that $\Psi$ is additive and fully faithful.

Theorem 1.25. The functor $\Psi: \operatorname{Bar}_{\gamma} \rightarrow \operatorname{Mod}_{\mathbb{R c}, \gamma^{\circ a}}\left(\mathbf{k}_{\mathbb{R}}\right)$ is an equivalence of additive categories.

Proof. By Theorem 1.17, there exist a locally finite family of intervals $\left\{I_{\alpha}\right\}_{\alpha \in A}$ such that $F \simeq \bigoplus_{\alpha \in A} \mathbf{k}_{I_{\alpha}}$. Since $\mu \operatorname{supp}\left(\mathbf{k}_{I_{\alpha}}\right) \subset \mathbb{R} \times \gamma^{\circ a}$, the intervals $I_{\alpha}$ are of the type $[a, b[$ with $-\infty \leq a<b \leq+\infty$.

Note that

$$
\operatorname{supp}(\Psi(A, I))=\operatorname{supp}(A, I) \quad \text { for a barcode }(A, I) \text {. }
$$

Let us denote by $\Psi^{-1}$ a quasi-inverse of the functor $\Psi$.

Definition 1.26. The barcode functor $\operatorname{Mod}_{\mathbb{R} c, \gamma^{\circ a}}\left(\mathbf{k}_{\mathbb{R}}\right) \stackrel{\sim}{\longrightarrow} \operatorname{Bar}_{\gamma}$ is the functor $\Psi^{-1}$.

Definition 1.26 extends to the derived category. Denote by $H^{\bullet}$ the functor $F \mapsto$ $\bigoplus_{j} H^{j}(F)$ from $\mathrm{D}^{\mathrm{b}}\left(\mathbf{k}_{\mathbb{R}}\right)$ to $\left(\operatorname{Mod}\left(\mathbf{k}_{\mathbb{R}}\right)\right)^{(\mathbb{Z})}$. One still calls

$$
\Psi^{-1} \circ H^{\bullet}: \mathrm{D}_{\mathbb{R} c, \gamma^{\circ \mathrm{a}}}^{\mathrm{b}}\left(\mathbf{k}_{\mathbb{R}}\right) \rightarrow \operatorname{Bar}_{\gamma}^{(\mathbb{Z})}
$$

the barcode functor. 
Example 1.27. Let $M$ be a real analytic manifold endowed with a subanalytic distance $d$, let $K \subset M$ be a compact subanalytic subset and let $f(x)=d(x, K)$. Then

$$
\Gamma_{f}^{+}=\{(x, t) ; d(x, K) \leq t\}
$$

and $f$ satisfies hypothesis 1.6. The barcodes of $K$ are those of $\mathrm{R} f_{*} \mathbf{k}_{\Gamma_{f}^{+}}$, that is, the image of $\mathrm{R} f_{*} \mathrm{k}_{\Gamma_{f}^{+}}$by the functor $\Psi^{-1} \circ H^{\bullet}$ of Definition 1.26 .

A natural question is to extend the definition of barcodes to $\mathbb{R}^{n}$ when $n>1$. For that purpose, it is natural to replace the cone $\{t \leq 0\}$ of $\mathbb{R}$ with a closed convex proper cone $\gamma$ of $\mathbb{V}$ as in subsection 1.2.

\section{Sheaves on vector spaces}

In this section, we denote by $\mathbb{V}$ a real vector space of finite dimension $n$.

We endow $\mathbb{V}$ with a Euclidean structure and denote by $\|\cdot\|$ the norm on $\mathbb{V}$. Let us denote by $B_{a}$ the closed ball with center the origin and radius $a \geq 0$ :

$$
B_{a}:=\{x \in \mathbb{V} ;\|x\| \leq a\}
$$

\subsection{Convolution}

References for this subsection are made to [Tam08, GS14].

Consider the maps

$$
\begin{aligned}
& s: \mathbb{V} \times \mathbb{V} \rightarrow \mathbb{V}, \quad s(x, y)=x+y, \\
& q_{i}: \mathbb{V} \times \mathbb{V} \rightarrow \mathbb{V}(i=1,2), \quad q_{1}(x, y)=x, q_{2}(x, y)=y .
\end{aligned}
$$

Recall that $a: \mathbb{V} \rightarrow \mathbb{V}$ denotes the antipodal map, $x \mapsto-x$. For a sheaf $F$, we set for short $F^{a}=a^{-1} F$.

One defines the convolution functor $\star: \mathrm{D}^{\mathrm{b}}\left(\mathbf{k}_{\mathbb{V}}\right) \times \mathrm{D}^{\mathrm{b}}\left(\mathbf{k}_{\mathbb{V}}\right) \rightarrow \mathrm{D}^{\mathrm{b}}\left(\mathbf{k}_{\mathbb{V}}\right)$ and its adjoint $\mathscr{H}_{o m^{\star}}:\left(\mathrm{D}^{\mathrm{b}}\left(\mathbf{k}_{\mathbb{V}}\right)\right)^{\mathrm{op}} \times \mathrm{D}^{\mathrm{b}}\left(\mathbf{k}_{\mathbb{V}}\right) \rightarrow \mathrm{D}^{\mathrm{b}}\left(\mathbf{k}_{\mathbb{V}}\right)$ by the formulas:

$$
\begin{aligned}
F \star G & :=\operatorname{R} s_{!}(F \otimes G), \\
\mathscr{H}_{o m^{\star}}(F, G) & :=\operatorname{R} q_{1 *} \operatorname{R} \mathscr{H} o m\left(q_{2}^{-1} G, s^{!} F\right) \\
& \simeq \operatorname{R} s_{*} \operatorname{R} \mathscr{H} o m\left(q_{2}^{-1}\left(G^{a}\right), q_{1}^{!} F\right) .
\end{aligned}
$$

For $F_{1}, F_{2}, F_{3} \in \mathrm{D}^{\mathrm{b}}\left(\mathbf{k}_{\mathbb{V}}\right)$, we have

$$
\operatorname{RHom}\left(F_{1} \star F_{2}, F_{3}\right) \simeq \operatorname{RHom}\left(F_{1}, \mathscr{H}_{o m}{ }^{\star}\left(F_{2}, F_{3}\right)\right) .
$$

With $\star$ as a tensor product, $\mathrm{D}^{\mathrm{b}}\left(\mathbf{k}_{\mathbb{V}}\right)$ is a commutative monoidal category with a unit object $\mathbf{k}_{\{0\}}: \mathbf{k}_{\{0\}} \star F \simeq F$ functorially in $F \in \mathrm{D}^{\mathrm{b}}\left(\mathbf{k}_{\mathbb{V}}\right)$. 
The functors $\star$ and $\mathscr{H} o m^{\star}$ induce functors (see [GS14, Cor. 3.1.4])

$$
\begin{aligned}
\cdot \star \bullet & : \mathrm{D}^{\mathrm{b}}\left(\mathbf{k}_{\mathbb{V}}\right) \times \mathrm{D}_{\gamma^{\circ a}}^{\mathrm{b}}\left(\mathbf{k}_{\mathbb{V}}\right) \rightarrow \mathrm{D}_{\gamma^{\circ a}}^{\mathrm{b}}\left(\mathbf{k}_{\mathbb{V}}\right), \\
\mathscr{H} o m^{\star}(\cdot, \cdot): & \left(\mathrm{D}^{\mathrm{b}}\left(\mathbf{k}_{\mathbb{V}}\right)\right)^{\mathrm{op}} \times \mathrm{D}_{\gamma^{\circ a}}^{\mathrm{b}}\left(\mathbf{k}_{\mathbb{V}}\right) \rightarrow \mathrm{D}_{\gamma^{\circ a}}^{\mathrm{b}}\left(\mathbf{k}_{\mathbb{V}}\right), \\
: & \left(\mathrm{D}_{\gamma^{\circ a}}^{\mathrm{b}}\left(\mathbf{k}_{\mathbb{V}}\right)\right)^{\mathrm{op}} \times \mathrm{D}^{\mathrm{b}}\left(\mathbf{k}_{\mathbb{V}}\right) \rightarrow \mathrm{D}_{\gamma^{\circ a}}^{\mathrm{b}}\left(\mathbf{k}_{\mathbb{V}}\right) .
\end{aligned}
$$

We set $X=\mathbb{R} \times \mathbb{V}$ and denote by $t$ the coordinate on $\mathbb{R}$. Following [GKS12, Exa 3.10] we recall that there exists $K \in \mathrm{D}_{\mathbb{R c}}^{\mathrm{b}}\left(\mathbf{k}_{X}\right)$ unique up to isomorphism such that

$$
\begin{aligned}
& \mu \operatorname{supp}(K) \subset\left\{(t, x ; \tau, \xi) ; \tau=\|\xi\| \neq 0, x=-t \tau^{-1} \xi\right\} \cup\{\tau=\xi=0\}, \\
& \left.K\right|_{t=0} \simeq \mathbf{k}_{\{0\}} .
\end{aligned}
$$

Moreover, there is a distinguished triangle

$$
\mathbf{k}_{\{t<-\|x\|\}}[n] \rightarrow K \rightarrow \mathbf{k}_{\{\|x\| \leq t\}} \stackrel{+1}{\longrightarrow} .
$$

Set $K_{a}=\left.K\right|_{t=a}$. Hence

$$
K_{a} \simeq \begin{cases}\mathbf{k}_{\{\|x\| \leq a\}} & \text { for } a \geq 0, \\ \mathbf{k}_{\{\|x\|<-a\}}[n] & \text { for } a<0 .\end{cases}
$$

We can easily check the isomorphism

$$
K_{a} \star K_{b} \simeq K_{a+b} \text { for } a, b \in \mathbb{R} .
$$

Hence, $K_{a} \star$ is an auto-equivalence of the category $\mathrm{D}^{\mathrm{b}}\left(\mathbf{k}_{\mathbb{V}}\right)$ as well as of the category $\mathrm{D}_{\mathbb{R}, \gamma^{\circ a}}^{\mathrm{b}}\left(\mathbf{k}_{\mathbb{V}}\right)$. A quasi-inverse is given by $K_{-a^{\star}}$.

Note that

$$
\operatorname{Hom}\left(K_{a} \star F, G\right) \simeq \operatorname{Hom}\left(F, K_{-a} \star G\right)
$$

and thus

$$
K_{a} \star F \simeq \mathscr{H} o m^{\star}\left(K_{-a}, F\right) .
$$

For $c \geq 0$ we have a canonical morphism $K_{c} \rightarrow \mathbf{k}_{\{0\}}$, which induces canonical morphisms for $a \geq b$ :

$$
\begin{array}{cl}
\chi_{b, a}: & K_{a} \longrightarrow K_{b}, \\
\chi_{b, a} \star F: & K_{a} \star F \rightarrow K_{b} \star F .
\end{array}
$$

In particular, one has

$$
K_{a} \star F \stackrel{\chi_{0, a} \star F}{\longrightarrow} F \stackrel{\chi_{b, 0} \star F}{\longrightarrow} K_{b} \star F \quad \text { for } a \geq 0 \geq b .
$$

Moreover, one has (recall that $\mathrm{D}_{\mathbb{V}}$ denotes the duality functor, see (1.2)) :

$$
\mathrm{D}_{\mathbb{V}}\left(K_{a}\right) \simeq K_{-a} .
$$


Lemma 2.1. For $F \in \mathrm{D}^{\mathrm{b}}\left(\mathbf{k}_{\mathbb{V}}\right)$, one has

$$
\mathrm{D}_{\mathbb{V}}\left(K_{a} \star F\right) \simeq K_{-a} \star \mathrm{D}_{\mathbb{V}}(F) .
$$

Proof. One has

$$
\begin{aligned}
\mathrm{D}_{\mathbb{V}}\left(K_{a} \star F\right) & \simeq \operatorname{R} \mathscr{H} o m\left(\operatorname{R} s_{!}\left(K_{a} \otimes F\right), \omega_{\mathbb{V}}\right) \\
& \simeq \operatorname{R} s_{*} \operatorname{R} \mathscr{H} o m\left(K_{a} \otimes F, s^{!} \omega_{\mathbb{V}}\right) \\
& \simeq \operatorname{R} s_{*} \operatorname{R} \mathscr{H}_{o m}\left(K_{a} \otimes F, \omega_{\mathbb{V} \times \mathbb{V}}\right) \\
& \simeq \operatorname{R} s_{*}\left(\mathrm{D}_{\mathbb{V}}\left(K_{a}\right) \otimes \mathrm{D}_{\mathbb{V}} F\right) \simeq K_{-a} \star \mathrm{D}_{\mathbb{V}} F .
\end{aligned}
$$

Note that the last isomorphism follows from the fact that $K_{a}$ has a compact support.

\subsection{Distance and the stability theorem}

A distance on persistence modules was first introduced in $\left[\mathrm{CCSG}^{+} 09\right]$ (see also [CdSGO16]), generalized to higher dimension in [Les15, LW15] and adapted to sheaves in [Cur13]. This distance is based on an idea of thickening the open sets. Note that the idea of thickening constructible functions already appeared in [Sch91], a paper inspired by [GRS83]. However, the idea of a distance in this framework is new as well as the so-called stability results, meaning a control of the distance after direct images.

Here we also introduce a thickening of sheaves and a pseudo-distance for sheaves and we prove a stability result. Our construction is based on the convolution of sheaves in the derived setting and therefore our results are essentially of a different nature from those mentioned above.

Definition 2.2. Let $F, G \in \mathrm{D}^{\mathrm{b}}\left(\mathbf{k}_{\mathbb{V}}\right)$ and let $a \geq 0$. One says that $F$ and $G$ are $a$ isomorphic if there are morphisms $f: K_{a} \star F \rightarrow G$ and $g: K_{a} \star G \rightarrow F$ which satisfies the following compatibility conditions: the composition $K_{2 a} \star F \stackrel{K_{a} \star f}{\longrightarrow} K_{a} \star G \stackrel{g}{\longrightarrow} F$ coincides with the natural morphism $\chi_{0,2 a} \star F: K_{2 a} \star F \rightarrow F$ and the composition $K_{2 a} \star G \stackrel{K_{a} \star g}{\longrightarrow} K_{a} \star F \stackrel{f}{\longrightarrow} G$ coincides with the natural morphism $\chi_{0,2 a} \star G: K_{2 a} \star G \rightarrow$ $G$.

Note that if $F$ and $G$ are $a$-isomorphic, then they are $b$-isomorphic for any $b \geq a$.

One sets

$$
\operatorname{dist}(F, G)=\inf \left(\{+\infty\} \cup\left\{a \in \mathbb{R}_{\geq 0} ; F \text { and } G \text { are } a \text {-isomorphic }\right\}\right)
$$

and calls $\operatorname{dist}(\cdot, \cdot)$ the convolution distance.

Note that for $F, G, H \in \mathrm{D}^{\mathrm{b}}\left(\mathbf{k}_{\mathbb{V}}\right)$,

- $F$ and $G$ are 0-isomorphic if and only if $F \simeq G$,

- $\operatorname{dist}(F, G)=\operatorname{dist}(G, F)$,

- $\operatorname{dist}(F, G) \leq \operatorname{dist}(F, H)+\operatorname{dist}(H, G)$. 
Remark 2.3. We don't know if $F$ and $G$ are $a$-isomorphic when $\operatorname{dist}(F, G) \leq a$.

Example 2.4. Let $F \in \mathrm{D}^{\mathrm{b}}\left(\mathbf{k}_{\mathbb{V}}\right)$ and assume that $\operatorname{supp}(F) \subset B_{a}$. Set $L:=\mathrm{R} \Gamma(\mathbb{V} ; F)$. Recall that, for a closed subset $S$ of $\mathbb{V}$, one denotes by $L_{S} \in \mathrm{D}^{\mathrm{b}}\left(\mathbf{k}_{\mathbb{V}}\right)$ the constant sheaf with stalk $L$ on $S$ extended by 0 on $\mathbb{V} \backslash S$. Let $G:=L_{\{0\}}$. We have

$$
K_{a} \star G \simeq L_{B_{a}}
$$

Denote by $q: \mathbb{V} \rightarrow$ pt the unique map from $\mathbb{V}$ to pt. The morphism $q^{-1} \mathrm{R} q_{*} F \rightarrow F$ defines the map $L_{\mathbb{V}} \rightarrow F$ and $F$ being supported by $B_{a}$, we get the morphism $g: K_{a} \star G \simeq$ $L_{B_{a}} \rightarrow F$. On the other hand,we have $\left(K_{a} \star F\right)_{0} \simeq L$ which defines $f: K_{a} \star F \rightarrow G$. One easily checks that $f$ and $g$ satisfy the compatibility conditions in Definition 2.2. Therefore

$$
\operatorname{dist}\left(F, L_{\{0\}}\right) \leq a .
$$

In particular, a non-zero object can be $a$-isomorphic to the zero object.

Remark 2.5. (i) If $\operatorname{dist}(F, G)<+\infty$, then we have

$$
\mathrm{R} \Gamma(\mathbb{V} ; F) \simeq \mathrm{R} \Gamma(\mathbb{V} ; G) \text { and } \mathrm{R} \Gamma_{\mathrm{c}}(\mathbb{V} ; F) \simeq \mathrm{R} \Gamma_{\mathrm{c}}(\mathbb{V} ; G)
$$

This follows from $\mathrm{R} \Gamma\left(\mathbb{V} ; K_{a} \star F\right) \stackrel{\sim}{\sim} \Gamma(\mathbb{V} ; F)$ and $\mathrm{R} \Gamma_{\mathrm{c}}\left(\mathbb{V} ; K_{a} \star F\right) \stackrel{\mathrm{R}}{\sim} \Gamma_{\mathrm{c}}(\mathbb{V} ; F)$ for $a \geq 0$.

Together with Example 2.4, for $F, G \in \mathrm{D}^{\mathrm{b}}\left(\mathbf{k}_{\mathbb{V}}\right)$ with $\operatorname{supp}(F), \operatorname{supp}(G) \subset B_{a}$, the condition $\operatorname{dist}(F, G) \leq 2 a$ holds if and only if $\mathrm{R} \Gamma(\mathbb{V} ; F) \simeq \mathrm{R} \Gamma(\mathbb{V} ; G)$.

(ii) Let $A$ and $B$ be closed convex subsets of $\mathbb{V}$. Then $\operatorname{dist}\left(\mathbf{k}_{A}, \mathbf{k}_{B}\right) \leq a$ if and only if $A \subset B+B_{a}$ and $B \subset A+B_{a}$.

Indeed, we may assume that $A$ and $B$ are non-empty. If $\mathbf{k}_{A}$ and $\mathbf{k}_{B}$ are $a$ isomorphic, then there exists a non-zero morphism $\mathbf{k}_{A+B_{a}} \simeq K_{a} \star \mathbf{k}_{A} \rightarrow \mathbf{k}_{B}$. Hence, we have $A+B_{a} \supset B$. Similarly we have $B+B_{a} \supset A$. The converse implication can be proved similarly.

(iii) Let $\gamma$ and $\gamma^{\prime}$ be closed convex cones. If $\operatorname{dist}\left(\mathbf{k}_{\gamma}, \mathbf{k}_{\gamma^{\prime}}\right)<+\infty$, then one has $\gamma=\gamma^{\prime}$. This follows from (ii).

(iv) Let $U$ and $V$ be open convex subsets of $\mathbb{V}$. Then $\operatorname{dist}\left(\mathbf{k}_{U}, \mathbf{k}_{V}\right) \leq a$ if and only if $U \subset V+B_{a}$ and $V \subset U+B_{a}$.

This follows from (ii), $\mathrm{D}_{\mathbb{V}}\left(\mathbf{k}_{U}[n]\right) \simeq \mathbf{k}_{\bar{U}}, \mathrm{D}_{\mathbb{V}}\left(\mathbf{k}_{\bar{U}}\right) \simeq \mathbf{k}_{U}[n]$ and Proposition 2.6 (i) below.

(v) Let $a \in \mathbb{R}$ and recall that the object $K_{a}$ satisfies (2.2). Using the isomorphism (2.3), one easily checks that for $a \geq 0$ one has

$$
\operatorname{dist}\left(K_{a}, K_{0}\right) \leq a, \operatorname{dist}\left(K_{-a}, K_{0}\right) \leq a .
$$

Since $K_{0} \simeq \mathbf{k}_{\{0\}}$ and $K_{-a}$ is the $n$-shifted constant sheaf on the open ball with radius $a$, we get an example of two sheaves concentrated in different degrees whose distance is finite. 
Proposition 2.6. (i) If $\operatorname{dist}(F, G) \leq a$, then $\operatorname{dist}\left(\mathrm{D}_{\mathbb{V}}(F), \mathrm{D}_{\mathbb{V}}(G)\right) \leq a$.

(ii) Assume that $\operatorname{dist}\left(F_{i}, G_{i}\right) \leq a_{i}(i=1,2)$. Then one has $\operatorname{dist}\left(F_{1} \star F_{2}, G_{1} \star G_{2}\right) \leq a_{1}+a_{2} \quad$ and $\operatorname{dist}\left(\mathscr{H}_{o m}{ }^{\star}\left(F_{1}, F_{2}\right), \mathscr{H} o m^{\star}\left(G_{1}, G_{2}\right)\right) \leq a_{1}+a_{2}$.

(iii) $\operatorname{dist}\left(\varphi_{\gamma}^{-1} \mathrm{R} \varphi_{\gamma_{*}} F, \varphi_{\gamma}^{-1} \mathrm{R} \varphi_{\gamma_{*}} G\right) \leq \operatorname{dist}(F, G)$.

Proof. (i) follows from Lemma 2.1.

(ii) Let $f_{i}: K_{a_{i}} \star F_{i} \rightarrow G_{i}(i=1,2)$. We get a map

$$
f_{1} \star f_{2}: K_{a_{1}} \star F_{1} \star K_{a_{2}} \star F_{2} \rightarrow G_{1} \star G_{2}
$$

and $K_{a_{1}} \star F_{1} \star K_{a_{2}} \star F_{2} \simeq K_{a_{1}+a_{2}} \star\left(F_{1} \star F_{2}\right)$. The end of the proof is straightforward and the case of $\mathscr{H}_{o m}^{\star}$ is similar.

(iii) follows from $\varphi_{\gamma}^{-1} \mathrm{R} \varphi_{\gamma_{*}} F \simeq \mathrm{R}_{*}\left(\mathbf{k}_{\gamma^{a}} \otimes F\right) \simeq \mathscr{H} O m^{\star}\left(\mathbf{k}_{\operatorname{Int}(\gamma)}[n], F\right)$ and (ii).

For a set $X$ and a map $f: X \rightarrow \mathbb{V}$, one sets

$$
\|f\|=\sup _{x \in X}\|f(x)\| \text {. }
$$

Theorem 2.7 (The stability theorem). Let $X$ be a locally compact space and let $f_{1}, f_{2}: X \rightarrow \mathbb{V}$ be two continuous maps. Then, for any $F \in \mathrm{D}^{\mathrm{b}}\left(\mathbf{k}_{X}\right)$, we have

$$
\operatorname{dist}\left(\mathrm{R} f_{1 *} F, \mathrm{R} f_{2 *} F\right) \leq\left\|f_{1}-f_{2}\right\| \quad \text { and } \operatorname{dist}\left(\mathrm{R} f_{1 !} F, \mathrm{R} f_{2 !} F\right) \leq\left\|f_{1}-f_{2}\right\| \text {. }
$$

Proof. (i) Since the proofs for $\mathrm{R} f_{*}$ and $\mathrm{R} f_{\text {! }}$ are similar, we shall only treat the case of $\mathrm{R} f_{*}$.

(ii) Set $a=\left\|f_{1}-f_{2}\right\|$. Let us show that $\mathrm{R} f_{1_{*}} F$ and $\mathrm{R} f_{2_{*}} F$ are $a$-isomorphic. Let us introduce some notations. Let $p_{1}: X \times \mathbb{V} \rightarrow X$ and $p_{2}: X \times \mathbb{V} \rightarrow \mathbb{V}$ be the projections. We set

$$
\begin{aligned}
G_{i} & =\left\{\left(x, f_{i}(x)\right) \in X \times \mathbb{V} ; x \in X\right\}, \\
S_{i} & =\left\{(x, y) \in X \times \mathbb{V} ;\left\|y-f_{i}(x)\right\| \leq a\right\}, \\
S_{i}^{\prime} & =\left\{(x, y) \in X \times \mathbb{V} ;\left\|y-f_{i}(x)\right\| \leq 2 a\right\} .
\end{aligned}
$$

for $i=1,2$. Then we have

$$
\begin{aligned}
\mathrm{R} f_{i_{*}} F & \simeq \mathrm{R} p_{2 *}\left(\mathbf{k}_{G_{i}} \otimes p_{1}^{-1} F\right), \\
K_{a} \star \mathrm{R} f_{i_{*}} F & \simeq \mathrm{R} p_{2_{*}}\left(\mathbf{k}_{S_{i}} \otimes p_{1}^{-1} F\right), \\
K_{2 a} \star \mathrm{R} f_{i_{*}} F & \simeq \mathrm{R} p_{2_{*}}\left(\mathbf{k}_{S_{i}^{\prime}} \otimes p_{1}^{-1} F\right) .
\end{aligned}
$$

Then $G_{2} \subset S_{1}$ induces a morphism $\mathbf{k}_{S_{1}} \rightarrow \mathbf{k}_{G_{2}}$, which induces $K_{a} \star \mathrm{R} f_{1 *} F \rightarrow \mathrm{R} f_{2 *} F$. Similarly, we construct $K_{a} \star \mathrm{R} f_{2 *} F \rightarrow \mathrm{R} f_{1 *} F$. Then, the composition

$$
K_{2 a} \star \mathrm{R} f_{1 *} F \rightarrow K_{a} \star \mathrm{R} f_{2 *} F \rightarrow \mathrm{R} f_{1 *} F
$$

is the canonical one, since these morphisms are induced by

$$
\mathbf{k}_{S_{1}^{\prime}} \rightarrow \mathbf{k}_{S_{2}} \rightarrow \mathbf{k}_{G_{1}} \text {. }
$$

The same argument holds when exchanging $f_{1}$ and $f_{2}$. 


\subsection{PL-sheaves}

Definition 2.8. A convex polytope $P$ in $\mathbb{V}$ is the intersection of a finite family of open or closed affine half-spaces.

From now on, we shall also assume that $\gamma$ is polyhedral, that is,

(2.8) $\gamma$ is a closed convex proper polyhedral cone with non-empty interior.

Definition 2.9. One says that $F \in \mathrm{D}_{\mathbb{R}_{c}}^{\mathrm{b}}\left(\mathbf{k}_{\mathbb{V}}\right)$ is $\mathrm{PL}$ (piecewise linear) if there exists a locally finite family $\left\{P_{a}\right\}_{a \in A}$ of convex polytopes such that $\mathbb{V}=\bigcup_{a \in A} P_{a}$ and $\left.F\right|_{P_{a}}$ is constant for any $a \in A$.

We shall use the notations (for a cone $\gamma$ satisfying (2.8)):

$$
\left\{\begin{array}{l}
\mathrm{D}_{\mathrm{PL}}^{\mathrm{b}}\left(\mathbf{k}_{\mathbb{V}}\right):=\left\{F \in \mathrm{D}_{\mathbb{R}_{\mathbb{c}}}^{\mathrm{b}}\left(\mathbf{k}_{\mathbb{V}}\right) ; F \text { is } \mathrm{PL}\right\} \\
\mathrm{D}_{\mathrm{PL}, \gamma^{\circ a}}^{\mathrm{b}}\left(\mathbf{k}_{\mathbb{V}}\right):=\mathrm{D}_{\mathrm{PL}}^{\mathrm{b}}\left(\mathbf{k}_{\mathbb{V}}\right) \cap \mathrm{D}_{\gamma^{\circ a}}^{\mathrm{b}}\left(\mathbf{k}_{\mathbb{V}}\right), \\
\operatorname{Mod}_{\mathrm{PL}}\left(\mathbf{k}_{\mathbb{V}}\right):=\operatorname{Mod}_{\mathbb{R c}}\left(\mathbf{k}_{\mathbb{V}}\right) \cap \mathrm{D}_{\mathrm{PL}}^{\mathrm{b}}\left(\mathbf{k}_{\mathbb{V}}\right), \\
\operatorname{Mod}_{\mathrm{PL}, \gamma^{\circ \mathrm{a}}}\left(\mathbf{k}_{\mathbb{V}}\right):=\operatorname{Mod}_{\mathrm{PL}}\left(\mathbf{k}_{\mathbb{V}}\right) \cap \operatorname{Mod}_{\gamma^{\circ a}}\left(\mathbf{k}_{\mathbb{V}}\right) .
\end{array}\right.
$$

Since the following result is easy to prove, we omit the proof.

Theorem 2.10. The category $\mathrm{D}_{\mathrm{PL}}^{\mathrm{b}}\left(\mathbf{k}_{\mathbb{V}}\right)$ is triangulated. Moreover:

(i) If $F$ and $F^{\prime}$ are $\mathrm{PL}$, then so are $F \otimes F^{\prime}$ and $\mathrm{R} \mathscr{H}$ om $\left(F, F^{\prime}\right)$.

(ii) Let $f: \mathbb{V} \rightarrow \mathbb{V}^{\prime}$ be a linear map.

(a) If $F^{\prime}$ is a PL sheaf on $\mathbb{V}^{\prime}$, then $f^{-1} F^{\prime}$ is a PL sheaf on $\mathbb{V}$.

(b) If $F$ is a $\mathrm{PL}$ sheaf on $\mathbb{V}$ and $\operatorname{supp}(F)$ is proper over $\mathbb{V}^{\prime}$, then $\mathrm{R} f_{*} F$ is a $\mathrm{PL}$ sheaf on $\mathbb{V}^{\prime}$.

(iii) Assume (2.8). If $F$ is $\mathrm{PL}$ and $\operatorname{supp}(F)$ is $\gamma$-proper (see Definition 1.3), then $\varphi_{\gamma}^{-1} \mathrm{R} \varphi_{\gamma_{*}} F$ is PL.

\subsection{The approximation theorem}

Theorem 2.11 (The approximation theorem). Let $F \in \mathrm{D}_{\mathbb{R} c}^{\mathrm{b}}\left(\mathbf{k}_{\mathbb{V}}\right)$. For each $\varepsilon>0$ there exists $G \in \mathrm{D}_{\mathrm{PL}}^{\mathrm{b}}\left(\mathbf{k}_{\mathbb{V}}\right)$ such that $\operatorname{dist}(F, G) \leq \varepsilon$ and $\operatorname{supp}(G) \subset \operatorname{supp}(F)+B_{\varepsilon}$.

Proof. We shall follow the notations of $[\mathrm{KS} 90, \S 8.1]$. Recall that a simplicial complex $\mathbf{S}=(S, \Delta)$ is the data consisting of a set $S$ and a set $\Delta$ of subsets of $S$, satisfying certain conditions (see loc. cit. Def. 8.1.1). For $\sigma \in \Delta$, one sets

$$
|\sigma|=\left\{(x(p))_{p \in S} \in \mathbb{R}_{\geq 0}^{S} ; \sum_{p \in S} x(p)=1, x(p)=0 \text { for } p \notin \sigma \text { and } x(p)>0 \text { for } p \in \sigma\right\}
$$


Note that the $|\sigma|$ 's are disjoint to each other. One also sets

$$
|\mathbf{S}|=\bigcup_{\sigma \in \Delta}|\sigma|
$$

We endow $|\mathbf{S}|$ with the induced topology from $\mathbb{R}_{\geq 0}^{S}$.

There exist a simplicial complex $\mathbf{S}=(S, \Delta)$ and a homeomorphism $f:|\mathbf{S}| \stackrel{\sim}{\longrightarrow}$ such that

$$
\left.\left(f^{-1} F\right)\right|_{|\sigma|} \text { is constant for any } \sigma \in \Delta \text {. }
$$

Replacing $\mathbf{S}$ with its successive barycentric subdivisions, we may assume further that $\|f(x)-f(y)\| \leq \varepsilon$ for any $\sigma \in \Delta$ and $x, y \in|\sigma|$. Then we set

$$
g(x)=\sum_{p \in S} x(p) f(p) .
$$

Here $S$ is identified with a subset of $|\mathbf{S}|$ by $S \ni q \mapsto x(p)=\delta_{p, q} \in \mathbb{R}^{S}$.

The map $g:|\mathbf{S}| \rightarrow \mathbb{V}$ is piecewise linear and continuous and satisfies:

$$
\|f(x)-g(x)\| \leq \varepsilon
$$

Hence $g$ is a proper map. Now we set $G=\mathrm{R} g_{*} f^{-1} F$. Then $\operatorname{supp}(G) \subset \operatorname{supp}(F)+B_{\varepsilon}$ and $G \in \mathrm{D}_{\mathrm{PL}}^{\mathrm{b}}\left(\mathbf{k}_{\mathbb{V}}\right)$, and since $F \simeq \mathrm{R} f_{*} f^{-1} F$, we have by Theorem 2.7

$$
\operatorname{dist}(F, G) \leq \varepsilon
$$

One can approximate any $\gamma$-sheaf with a PL- $\gamma$-sheaf. Indeed:

Corollary 2.12. Assume (2.8). Let $F \in \mathrm{D}_{\mathbb{R} \text {, } \gamma^{\text {oa }}}^{\mathrm{b}}\left(\mathbf{k}_{\mathbb{V}}\right)$ such that $\operatorname{supp}(F)$ is $\gamma$-proper. For each $\varepsilon>0$ there exists $G \in \mathrm{D}_{\mathrm{PL}, \gamma^{\circ a}}^{\mathrm{b}}\left(\mathbf{k}_{\mathbb{V}}\right)$ such that $\operatorname{dist}(F, G) \leq \varepsilon$.

Proof. We shall apply Theorem 2.11. There exists $G \in \mathrm{D}_{\mathrm{PL}}^{\mathrm{b}}\left(\mathbf{k}_{\mathbb{V}}\right) \operatorname{such}$ that $\operatorname{dist}(F, G) \leq$ $\varepsilon$ and $\operatorname{supp}(G) \subset \operatorname{Supp}(F)+B_{\varepsilon}$. Hence $\operatorname{supp}(G)$ is $\gamma$-proper. Then, $\varphi_{\gamma}^{-1} \mathrm{R} \varphi_{\gamma_{*}} G \in$ $\mathrm{D}_{\mathrm{PL}, \gamma^{\circ a}}^{\mathrm{b}}\left(\mathbf{k}_{\mathbb{V}}\right)$ by Theorem 2.10. On the other hand, Proposition 2.6 (iii) implies that

$$
\operatorname{dist}\left(\varphi_{\gamma}^{-1} \mathrm{R} \varphi_{\gamma_{*}} F, \varphi_{\gamma}^{-1} \mathrm{R} \varphi_{\gamma_{*}} G\right) \leq \operatorname{dist}(F, G) \leq \varepsilon
$$

Since $F$ is a $\gamma$-sheaf, one has $\varphi_{\gamma}^{-1} \mathrm{R} \varphi_{\gamma_{*}} F \simeq F$.

\subsection{Barcodes (multi-dimensional case)}

Recall that a family of subsets $Z=\left\{Z_{\alpha}\right\}_{\alpha \in A}$ is locally finite if for any compact subset $K$ of $\mathbb{V}$, the set $\left\{\alpha \in A ; Z_{\alpha} \cap K \neq \varnothing\right\}$ is finite. 
Definition 2.13. Assume (2.8). A $\gamma$-barcode $(A, Z)$ in $\mathbb{V}$, or simply, a barcode, is the data of a set of indices $A$ and a family $Z=\left\{Z_{\alpha}\right\}_{\alpha \in A}$ of subsets of $\mathbb{V}$, these data satisfying
$\left\{\right.$ (i) the family $Z=\left\{Z_{\alpha}\right\}_{\alpha \in A}$ is locally finite in $\mathbb{V}$,
(ii) the $Z_{\alpha}$ 's are non-empty, $\gamma$-locally closed, convex polytopes.

The support of the $\gamma$-barcode $(A, Z)$, denoted by $\operatorname{supp}(A, Z)$, is the set $\bigcup_{\alpha \in A} \overline{Z_{\alpha}}$.

Let us say that a barcode $(A, Z)$ is elementary if $A \simeq$ pt. We shall identify the elementary barcode (pt, $Z$ ) with the $\gamma$-locally closed convex polytope $Z$.

The barcodes are the objects of the additive category $\mathbf{B a r}_{\gamma}$ that we shall describe now.

- the zero-barcode is $0=(A, Z)$ with $A=\varnothing$,

- for two barcodes $(A, S)$ and $(B, Z)$, we set $(A, S) \oplus(B, Z)=(A \sqcup B, S \sqcup Z)$. In other words, the sum of $\left\{S_{\alpha}\right\}_{\alpha \in A}$ and $\left\{Z_{\beta}\right\}_{\beta \in B}$ is the barcode $\left\{W_{\gamma}\right\}_{\gamma \in C}$ with $C=A \sqcup B$ and $W_{\gamma}=S_{\alpha}$ or $W_{\gamma}=Z_{\beta}$ according as $\gamma=\alpha \in A$ or $\gamma=\beta \in B$.

- For two elementary barcodes $S$ and $T$ one sets

(2.11)Hom Bar $_{\gamma}(S, T)= \begin{cases}\mathbf{k} & \text { if } S \cap T \text { is non-empty, closed in } S \text { and open in } T \\ 0 & \text { otherwise. }\end{cases}$

Note that

$$
\operatorname{Hom}_{\mathbf{B a r}_{\gamma}}(S, T) \simeq \operatorname{Hom}\left(\mathbf{k}_{S}, \mathbf{k}_{T}\right)
$$

- One extends this construction to barcodes by linearity. For two barcodes $(A, S)=$ $\left\{S_{\alpha}\right\}_{\alpha \in A}$ and $(B, T)=\left\{T_{\beta}\right\}_{\beta \in B}$, one sets

$$
\begin{aligned}
\operatorname{Hom}_{\mathbf{B a r}_{\gamma}}((A, S),(B, T)) & =\prod_{\alpha \in A, \beta \in B} \operatorname{Hom}_{\mathbf{B a r}_{\gamma}}\left(S_{\alpha}, T_{\beta}\right) \\
& \simeq \operatorname{Hom}\left(\bigoplus_{\alpha \in A} \mathbf{k}_{S_{\alpha}}, \prod_{\beta \in B} \mathbf{k}_{T_{\beta}}\right) \\
& \simeq \operatorname{Hom}\left(\bigoplus_{\alpha \in A} \mathbf{k}_{S_{\alpha}}, \bigoplus_{\beta \in B} \mathbf{k}_{T_{\beta}}\right) .
\end{aligned}
$$

(The last isomorphism follows from the fact that the sum is locally finite, similarly to the one-dimension case.) The composition of $u \in \operatorname{Hom}_{\mathbf{B a r}_{\gamma}}((A, S),(B, T))$ and $v \in \operatorname{Hom}_{\mathbf{B a r}_{\gamma}}((B, T),(C, V))$ is defined similarly to the one-dimension case (see (1.13)), or by using (2.13).

There is a natural functor

$$
\Psi: \operatorname{Bar}_{\gamma} \rightarrow \operatorname{Mod}_{\mathrm{PL}, \gamma^{\mathrm{oa}}}\left(\mathbf{k}_{\mathbb{V}}\right), \quad Z=\left\{Z_{\alpha}\right\}_{\alpha \in A} \mapsto \bigoplus_{\alpha \in A} \mathbf{k}_{Z_{\alpha}}
$$

and the functor $\Psi$ is fully faithful in view of (2.13). We have seen that when $\operatorname{dim} \mathbb{V}=1$ the functor $\Psi$ is an equivalence of categories. However, the functor $\Psi$ is no more essentially surjective when $\operatorname{dim} \mathbb{V}>1$ as seen in the following examples. 
Example 2.14. Denote by $(x, y)$ the coordinates on $\mathbb{R}^{2}$ and consider the sets

$$
\begin{aligned}
& \gamma=\{(x, y) ; x \leq 0, y \leq 0\}, \\
& A=(1,0)+\gamma^{a}, \quad B=(0,1)+\gamma^{a}, \quad C=(2,2)+\gamma^{a} .
\end{aligned}
$$

Define the $\gamma$-sheaf $F$ by the exact sequence $0 \rightarrow F \rightarrow \mathbf{k}_{A} \oplus \mathbf{k}_{B} \rightarrow \mathbf{k}_{C} \rightarrow 0$. Then $F \simeq \mathbf{k}_{A} \oplus \mathbf{k}_{B}$ on $\mathbb{R}^{2} \backslash C$ and $F$ has rank one on $\operatorname{Int}(C)$.

Let us show that $\operatorname{End}_{\operatorname{Mod}_{\mathbb{R c}}\left(\mathbf{k}_{\mathbb{V}}\right)}(F) \simeq \mathbf{k}$.

Let $u$ and $v$ be the generators of $\Gamma\left(\mathbb{V} ; \mathbf{k}_{A}\right)$ and $\Gamma\left(\mathbb{V} ; \mathbf{k}_{B}\right)$, respectively. Then one has $F(\mathbb{V})=\mathbf{k}(u-v) \subset \Gamma\left(\mathbb{V} ; \mathbf{k}_{A} \oplus \mathbf{k}_{B}\right)$. We have an exact sequence

$$
0 \rightarrow \mathbf{k}_{A \backslash B} \rightarrow \mathbf{k}_{A \cup B} \oplus \mathbf{k}_{A \backslash C} \rightarrow F \rightarrow 0 .
$$

Here, the composition $\mathbf{k}_{A \backslash C} \rightarrow F \rightarrow \mathbf{k}_{B}$ vanishes and the morphism $\mathbf{k}_{A \cup B} \rightarrow F$ is given by $u-v$. Let $f \in \operatorname{End}(F)$. We shall show $f \in \mathbf{k i d}_{F}$. We may assume that $f(u-v)=0$ from the beginning, i.e., the composition $g: \mathbf{k}_{A \cup B} \rightarrow F \stackrel{f}{\longrightarrow} F$ vanishes. Then the composition $\mathbf{k}_{A \backslash C} \rightarrow F \stackrel{f}{\longrightarrow} F \rightarrow \mathbf{k}_{A}$ vanishes, since it coincides with $g$ on $A \backslash B$. The composition $\mathbf{k}_{A \backslash C} \rightarrow F \stackrel{f}{\longrightarrow} F \rightarrow \mathbf{k}_{B}$ vanishes since Hom $\left(\mathbf{k}_{A \backslash C}, \mathbf{k}_{B}\right) \simeq 0$. Hence $f=0$.

Therefore $F$ is indecomposable. Hence, $F$ is not in the essential image of $\Psi$.

Example 2.15. Denote by $(x, y, z)$ the coordinates on $\mathbb{R}^{3}$ and consider the sets

$$
\begin{aligned}
& \gamma=\{(x, y, z) ; x \leq-(|y|+|z|)\}, \quad S=\{(x, y, z) ; x=0,|y|+|z|=1\} \\
& Z=\left(S+\gamma^{a}\right) \cap\{x<1\} .
\end{aligned}
$$

Then $Z$ is a $\gamma$-barcode. Since $Z \cap\{(x, y, z) ; y=z=0\}=\varnothing$, we consider the map $r: Z \rightarrow \mathbb{R}^{3} \backslash \mathbb{R} \times\{(0,0)\} \rightarrow \mathbb{R}^{2} \backslash\{(0,0)\} \rightarrow \mathbb{S}^{1}$. Then the composition $S \rightarrow Z \stackrel{r}{\rightarrow} \mathbb{S}^{1}$ is an isomorphism. Let $L$ be a locally constant but non constant sheaf of rank 1 on $\mathbb{S}^{1}$. Then, the sheaf $F=r^{-1} L$ is locally isomorphic to $\mathbf{k}_{Z}$ but $F$ is not isomorphic to $\mathbf{k}_{Z}$ since $r_{*}\left(F \otimes \mathbf{k}_{S}\right) \simeq L \not \mathbf{k}_{\mathbb{S}^{1}}$. Hence $F$ is not in the essential image of $\Psi$.

Note that a different definition of higher dimensional barcodes is proposed in [Cur13, Def. 8.3.4].

Definition 2.16. An object of $\operatorname{Mod}_{\mathrm{PL}, \gamma^{\circ a}}\left(\mathbf{k}_{\mathbb{V}}\right)$ is a barcode $\gamma$-sheaf if it is in the essential image of $\Psi$.

\section{$3 \quad$ Piecewise linear $\gamma$-sheaves}

The aim of this section is to prove Theorem 3.17.

\subsection{Complements on the $\gamma$-topology}

We denote by $\gamma$ a closed proper convex cone with non-empty interior, as in (1.3), and recall Definition 1.3. 


\section{Lemma 3.1.}

(i) for any subset $A$ of $\mathbb{V}$, one has $A+\operatorname{Int}(\gamma)=\bar{A}+\operatorname{Int}(\gamma)$.

(ii) If $U$ is an open subset of $\mathbb{V}$, then we have $U+\operatorname{Int}(\gamma)=U+\gamma$. In particular $U \subset U+\operatorname{Int}(\gamma)$

Proof. (i) For $x \in \bar{A}$ and $v \in \operatorname{Int}(\gamma)$, let us show $x+v \in A+\operatorname{Int}(\gamma)$. Since $x+v-\operatorname{Int}(\gamma)$ is a neighborhood of $x$, there exists $y \in A \cap(x+v-\operatorname{Int}(\gamma))$. Then, one has $x+v \in$ $y+\operatorname{Int}(\gamma) \subset A+\operatorname{Int}(\gamma)$.

(ii) Let us show $U \subset U+\operatorname{Int}(\gamma)$. For $x \in U$, there exists $y \in \operatorname{Int}(\gamma) \cap(x-U)$. Then $x \in y+U \subset U+\operatorname{Int}(\gamma)$.

To complete it is enough to remark that $U+\gamma \subset(U+\operatorname{Int}(\gamma))+\gamma=U+\operatorname{Int}(\gamma)$,

\section{Lemma 3.2.}

(a) The intersection of a $\gamma$-invariant set and a $\gamma^{a}$-invariant set is $\gamma$-flat, i.e., $(B+\gamma) \cap$ $\left(C+\gamma^{a}\right)$ is $\gamma$-flat for any $B, C \subset \mathbb{V}$.

(b) If $A$ is $\gamma$-flat, then $\operatorname{Int}(A)=(A+\operatorname{Int}(\gamma)) \cap\left(A+\operatorname{Int}\left(\gamma^{a}\right)\right)$ and $\operatorname{Int}(A)$ is $\gamma$-flat.

(c) If $U$ is $\gamma$-open, then one has $\operatorname{Int}(\bar{U})=U$.

(d) if $U$ is a $\gamma$-flat open subset of $\mathbb{V}$, then $Z:=(U+\gamma) \cap \overline{U+\gamma^{a}}$ is $\gamma$-locally closed and $\operatorname{Int}(Z)=U$.

Proof. (a) Set $D=(B+\gamma) \cap\left(C+\gamma^{a}\right)$. Then we have $D+\gamma \subset(B+\gamma)+\gamma=B+\gamma$. Similarly we have $D+\gamma^{a} \subset\left(C+\gamma^{a}\right)+\gamma^{a}=C+\gamma^{a}$. Hence, we obtain $(D+\gamma) \cap\left(D+\gamma^{a}\right) \subset$ $(B+\gamma) \cap\left(C+\gamma^{a}\right)=D$.

(b) Set $U=(A+\operatorname{Int}(\gamma)) \cap\left(A+\operatorname{Int}\left(\gamma^{a}\right)\right)$. Then $U$ is open and contained in $A$, hence, in $\operatorname{Int}(A)$. The other inclusion follows from Lemma 3.1 (ii).

Note that $\operatorname{Int}(A)$ is $\gamma$-flat by (a).

(c) Let $x \in \operatorname{Int}(\bar{U})$. Then there exists $v \in \operatorname{Int}\left(\gamma^{a}\right)$ such that $x+v \in \bar{U}$. Since $x+\operatorname{Int}\left(\gamma^{a}\right)$ is a neighborhood of $x+v$, there exists $y \in U \cap\left(x+\operatorname{Int}\left(\gamma^{a}\right)\right)$. Hence we have $x \in y+\operatorname{Int}(\gamma) \subset U$.

(d) It is obvious that $Z$ is $\gamma$-locally closed and $U \subset \operatorname{Int}(Z)$. Conversely, (c) implies that $\operatorname{Int}(Z) \subset(U+\gamma) \cap \operatorname{Int}\left(\overline{U+\gamma^{a}}\right)=(U+\gamma) \cap\left(U+\gamma^{a}\right)=U$.

Lemma 3.3. Let $Z$ be a $\gamma$-locally closed subset of $\mathbb{V}$ and $\Omega=\operatorname{Int}(Z)$. Then

(a) for any $x \in Z$, there exists a neighborhood $W$ of $x$ such that $\left(x+\gamma^{a}\right) \cap W \subset Z$,

(b) if $x \in \mathbb{V}$ satisfies $x \in \overline{\left(x+\gamma^{a}\right) \cap Z}$, then one has $x \in Z$,

(c) $Z$ is $\gamma$-flat,

(d) $\Omega+\operatorname{Int}(\gamma)=Z+\operatorname{Int}(\gamma)=Z+\gamma$,

(e) $Z=(\Omega+\gamma) \cap \overline{\Omega+\gamma^{a}}=(\Omega+\gamma) \cap \bar{\Omega}$, 
(f) $\Omega=(Z+\gamma) \cap\left(Z+\operatorname{Int}\left(\gamma^{a}\right)\right)=Z \cap\left(Z+\operatorname{Int}\left(\gamma^{a}\right)\right)$

$=\left\{x \in \mathbb{V} ; \begin{array}{l}\text { there exists an open neighborhood } W \text { of } x \\ \text { such }(x+\gamma) \cap W \subset Z\end{array}\right\}$,

(g) $\Omega$ is $\gamma$-flat.

Proof. Write $Z=A \cap B$ with a $\gamma$-open $A$ and $\gamma$-closed $B$. Hence $A+\gamma=A$ and $B+\gamma^{a}=B$.

(a) Setting $W=A$, one has $\left(x+\gamma^{a}\right) \cap W \subset B \cap A=Z$.

(b) Assume that $x \in \overline{\left(x+\gamma^{a}\right) \cap Z}$. Then one has $x \in \bar{Z} \subset B$. Hence it remains to show $x \in A$. Since $\left(x+\gamma^{a}\right) \cap Z \neq \varnothing$, there exists $y \in\left(x+\gamma^{a}\right) \cap Z$. Then one has $x \in y+\gamma \subset A+\gamma=A$.

(c) follows from Lemma 3.2 (a).

(d) It is enough to show that $Z \subset \Omega+\operatorname{Int}(\gamma)$. Let $x \in Z$. Then $A \cap\left(x+\operatorname{Int}\left(\gamma^{a}\right)\right) \subset$ $A \cap B=Z$, which implies that

$$
A \cap\left(x+\operatorname{Int}\left(\gamma^{a}\right)\right) \subset \Omega \text { for any } x \in Z .
$$

Since $A \cap\left(x+\operatorname{Int}(\gamma)^{a}\right) \neq \varnothing$, there exists $y \in A \cap\left(x+\operatorname{Int}(\gamma)^{a}\right)$. Then one has $x \in y+\operatorname{Int}(\gamma) \subset \Omega+\operatorname{Int}(\gamma)$.

(e) One has $(\Omega+\gamma) \cap \overline{\Omega+\gamma^{a}} \subset A \cap B=Z$. Let us show $Z \subset(\Omega+\gamma) \cap \bar{\Omega}$. The inclusion $Z \subset \Omega+\gamma$ follows from (d). Finally, let us show that $x \in \bar{\Omega}$ for any $x \in Z$. By (3.1), one has $x \in \overline{A \cap\left(x+\operatorname{Int}\left(\gamma^{a}\right)\right)} \subset \bar{\Omega}$.

(f) We shall show $(Z+\gamma) \cap\left(Z+\operatorname{Int}\left(\gamma^{a}\right)\right) \subset \Omega \subset Z \cap\left(Z+\operatorname{Int}\left(\gamma^{a}\right)\right)$. We have

$$
(Z+\gamma) \cap\left(Z+\operatorname{Int}\left(\gamma^{a}\right)\right) \subset(\Omega+\gamma) \cap\left(Z+\operatorname{Int}\left(\gamma^{a}\right)\right)=A \cap B=Z .
$$

Here the first inclusion follows from (d). Hence we obtain $(Z+\gamma) \cap\left(Z+\operatorname{Int}\left(\gamma^{a}\right)\right) \subset \Omega$.

The inclusion $\Omega \subset Z \cap\left(Z+\operatorname{Int}\left(\gamma^{a}\right)\right)$ follows from $\Omega \subset \Omega+\operatorname{Int}\left(\gamma^{a}\right)$, which is a consequence of Lemma 3.1 (ii).

Let us prove the last equality. Let $x \in \mathbb{V}$ such that $(x+\gamma) \cap W \subset Z$ for an open neighborhood $W$ of $x$. Take $y \in(x+\operatorname{Int}(\gamma)) \cap W \subset Z$. Then we have $x \in y+\operatorname{Int}\left(\gamma^{a}\right) \subset$ $Z+\operatorname{Int}\left(\gamma^{a}\right)$.

(g) One has

$$
(\Omega+\gamma) \cap\left(\Omega+\gamma^{a}\right)=(\Omega+\gamma) \cap\left(\Omega+\operatorname{Int}\left(\gamma^{a}\right)\right) \subset(\Omega+\gamma) \cap\left(Z+\operatorname{Int}\left(\gamma^{a}\right)\right) \subset \Omega .
$$

Here the first equality is by Lemma 3.1 (ii) and the last inclusion follows from (f).

The following proposition now follows from Lemma 3.2 and Lemma 3.3.

Proposition 3.4. The set of $\gamma$-flat open subsets $\Omega$ of $\mathbb{V}$ and the set of $\gamma$-locally closed subsets $Z$ of $\mathbb{V}$ are isomorphic by the correspondence

$$
\begin{aligned}
& \Omega \longmapsto \\
& \operatorname{Int}(Z)\longleftrightarrow Z+\gamma) \cap \overline{\Omega+\gamma^{a}} \\
& Z
\end{aligned}
$$


Lemma 3.5. Let $U_{i}(i=1,2)$ be $\gamma$-flat open subsets such that $U_{1} \cap U_{2}=\varnothing$. Set $Z_{i}=\left(U_{i}+\gamma\right) \cap \overline{U_{i}+\gamma^{a}}$. Then one has $Z_{1} \cap Z_{2}=\varnothing$.

Proof. Since $Z_{i}$ is $\gamma$-locally closed, $Z_{1} \cap Z_{2}$ is also $\gamma$-locally closed. Then, by Lemma 3.3, $Z_{1} \cap Z_{2}$ is contained in the closure of $\operatorname{Int}\left(Z_{1} \cap Z_{2}\right) \subset \operatorname{Int}\left(Z_{1}\right) \cap \operatorname{Int}\left(Z_{2}\right)=U_{1} \cap U_{2}=\varnothing$.

\subsection{Study of $\gamma$-sheaves}

In this subsection, we shall obtain some results on the behaviour of constructible $\gamma$ sheaves, preliminary to the study of piecewise linear $\gamma$-sheaves and Theorem 3.17.

Lemma 3.6. Let $M$ be a manifold and $N$ a submanifold of $M$ of codimension $\geq 2$. Let $\Lambda$ be a closed conic involutive subset of $T^{*} M$. If $\pi\left(\Lambda \cap \dot{T}^{*} M\right)=N$, then $T_{N}^{*} M \subset \Lambda$.

Proof. We choose a local coordinate system $(x)=(t, y)$ such that $N=\{t=0\}$, $(t)=\left(t_{1}, \ldots, t_{m}\right)$. Let $(x ; \xi)=(t, y ; \tau, \eta)$ denote the associated coordinates on $T^{*} M$. Let $\left(0, y_{0}\right) \in N$. There exists $\left(\tau_{0}, \eta_{0}\right) \neq 0$ such that $\left(0, y_{0} ; \tau_{0}, \eta_{0}\right) \in \Lambda$. Since $\Lambda \cap$ $\dot{T}^{*} M$ is contained in $t=0, \Lambda \cap \dot{T}^{*} M$ is invariant by $\frac{\partial}{\partial \tau_{k}}$ for $1 \leq k \leq m$ by [KS90, Proposition 6.5.2]. Since $m>1$, this implies that $\Lambda \cap \dot{T}^{*} M$ contains $\left(0, y_{0} ; \tau, \eta_{0}\right)$ for any $\tau$. Moreover, since $\Lambda$ is conic, this implies $\left(0, y_{0} ; \tau, 0\right) \in \Lambda \cap \dot{T}^{*} M$ for any $\tau$.

Proposition 3.7. Let $F \in \operatorname{Mod}_{\mathbb{R} c, \gamma^{\circ a}}\left(\mathbf{k}_{\mathbb{V}}\right)$. Then $S:=\operatorname{Sing}(F)$ has pure codimension 1 . Moreover, for any $x \in S_{\mathrm{reg}}$, one has $T_{x} S \cap \operatorname{Int}(\gamma)=\varnothing$, or equivalently $\left(T_{S}^{*} \mathbb{V}\right)_{x} \subset \gamma^{\circ} \cup \gamma^{\circ a}$.

Proof. Assume that there is a point where $S$ has codimension $\geq 2$. Take an open subset $U$ such that $S \cap U$ is a non-empty submanifold of codimension $\geq 2$. Note that $\Lambda:=\mu \operatorname{supp}(F)$ is involutive ([KS90, Theorem .6.5.4]), and $U \cap \pi\left(\Lambda \cap \dot{T}^{*} M\right)=S \cap U$. Hence Lemma 3.6 implies that $\pi^{-1} U \cap T_{S}^{*} M \subset \Lambda \subset \mathbb{V} \times \gamma^{\circ a}$. It contradicts the fact that $\gamma^{\circ a}$ is a proper closed convex cone.

The last assertion is a consequence of the fact that $\mu \operatorname{supp}(F) \cap \pi^{-1} U \subset T_{S_{\text {reg }}^{*}}^{*} \mathbb{V}$ for an open dense subanalytic subset $U$ of $S_{\text {reg }}$ and hence, $S$ being an hypersurface, $\left(T_{S_{\text {reg }}}^{*} \mathbb{V} \cap \pi^{-1} U\right) \subset \mu \operatorname{supp}(F) \cup \mu \operatorname{supp}(F)^{a}$.

From now on, and until the end of this paper, we assume that $\gamma$ satisfies (2.8).

Lemma 3.8. Assume (2.8). Let $x \in \mathbb{V}$, let $I$ be an open interval of $\mathbb{R}$ with $0 \in I$ and let $c: I \rightarrow \mathbb{V}$ be a real analytic map. Assume that $c(t) \in(x+\gamma) \backslash\{x\}$ for $t \in I, t>0$ and $c(0)=x$. Then $c^{\prime}(t) \in \gamma$ for all $t \geq 0$ in a neighborhood of 0 .

Proof. Since $\gamma$ is polyhedral, we may assume that $\gamma=\{x \in \mathbb{V} ; f(x) \geq 0\}$ for a linear function $f(x)$ on $\mathbb{V}$. Set $\varphi(t)=f(c(t))$. it is enough to prove that $\varphi^{\prime}(t) \geq 0$ for $t \geq 0$ in a neighborhood of 0 . If $\varphi=0$, the result is clear. Otherwise, there exists $m \in \mathbb{N}$, $m>0$ such that $\varphi(t)=t^{m} v+O\left(t^{m+1}\right)$ with $v \neq 0$. Then $v>0$ and it follows that $\varphi^{\prime}(t)>0$ for $t>0$ in a neighborhood of $t=0$.

Remark 3.9. Lemma 3.8 is no more true without the assumption that the cone is polyhedral. Consider the cone $\gamma=\left\{(x, y, z) \in \mathbb{R}^{3} ; x^{2}+y^{2} \leq z^{2}, z \geq 0\right\}$ and the curve $c(t)=(t \cos (t), t \sin (t), t)$. One easily checks that $c^{\prime}(t) \notin \gamma$ for $t>0$. 
Theorem 3.10. Assume (2.8). Let $F \in \mathrm{D}_{\mathbb{R c}, \gamma^{\circ \mathrm{a}}}^{\mathrm{b}}\left(\mathbf{k}_{\mathbb{V}}\right)$. Then for each $x \in \mathbb{V}$, there exists an open neighborhood $U$ of $x$ such that $\left.F\right|_{\left(x+\gamma^{a}\right) \cap U}$ is constant.

Proof. (i) By Corollary 1.7, we may assume that $F$ is concentrated in degree 0. Moreover, the sheaf $F \otimes \mathbf{k}_{x+\gamma^{a}}$ belongs to $\operatorname{Mod}_{\mathbb{R} \mathbf{c}, \gamma^{\circ a}}\left(\mathbf{k}_{\mathbb{V}}\right)$. Hence we may assume from the beginning that $\operatorname{supp}(F) \subset x+\gamma^{a}$.

(ii) By Theorem 1.5, for any $y \in \mathbb{V}, F(y+\gamma) \stackrel{\sim}{\longrightarrow} F_{y}$. Since $F$ is $\mathbb{R}$-constructible, there exists an open neighborhood $V$ of $x$ such that $\Gamma(V ; F) \stackrel{\sim}{\longrightarrow} F_{x}$. Let $y \in V \cap\left(x+\gamma^{a}\right)$. Then $x \in y+\gamma$. Consider the diagram

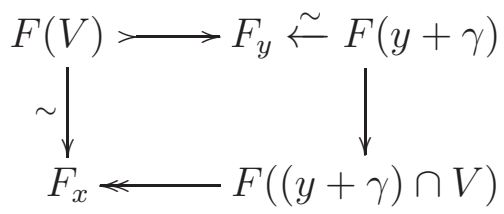

Let $E:=F_{x}$, a finite-dimensional $\mathbf{k}$-vector space. We have an injective map $E \rightarrow F_{y}$ for all $y \in\left(x+\gamma^{a}\right) \cap V$, hence a monomorphism $E_{\left(x+\gamma^{a}\right) \cap V} \hookrightarrow F$. Define $G$ as the cokernel of this map. Then $G_{x} \simeq 0, \operatorname{supp}(G) \subset x+\gamma^{a}$ and $G \in \operatorname{Mod}_{\mathbb{R c}, \gamma^{\circ a}}\left(\mathbf{k}_{\mathbb{V}}\right)$. It remains to show that $G \simeq 0$ in a neighborhood of $x$.

(iii) Assume that $G \neq 0$ in any neighborhood of $x$. Then $\left\{y \in \mathbb{V} ; G_{y} \neq 0\right\}$ is a subanalytic set whose closure contains $x$. By the curve selection lemma, we find an analytic curve $c: I \rightarrow \gamma^{a}$ such that $c(0)=x$ and $G_{c(t)} \neq 0$ for any $t \in I$ such that $t>0$. By Lemma 3.8, $c^{\prime}(t) \in \gamma^{a}$ for all $t \geq 0$ in a neighborhood of 0 . Setting $\varphi(t)=c\left(t^{2}\right)$ for $t \geq 0$ and $\varphi(t)=x$ for $t \leq 0$, we find a curve of class $C^{1}$ and $\operatorname{supp}\left(\varphi^{-1} G\right) \subset\{t \geq 0\}$. Denote by $(t ; \tau)$ the homogeneous symplectic coordinates on $T^{*} \mathbb{R}$. Applying [KS90, Cor. 6.4.4], we get

$$
\left\{\begin{array}{l}
(0 ; \tau) \in \mu \operatorname{supp}\left(\varphi^{-1} G\right) \text { implies that there exists a sequence }\left\{\left(x_{n} ; \xi_{n}\right)\right\}_{n} \subset \\
\operatorname{SS}(G) \text { with } x_{n} \stackrel{n}{\longrightarrow} x, t_{n} \stackrel{n}{\longrightarrow} 0,\left\langle\varphi^{\prime}\left(t_{n}\right), \xi_{n}\right\rangle \stackrel{n}{\longrightarrow} \tau .
\end{array}\right.
$$

Since $\varphi^{\prime}\left(t_{n}\right) \in \gamma^{a}$ and $\xi_{n} \in \gamma^{\circ a}$, we get $\tau \geq 0$. Hence, $\varphi^{-1} G \simeq 0$ in a neighborhood of 0 . This is a contradiction.

For any sheaf $F \in D_{\mathbb{R c}}^{\mathrm{b}}\left(\mathbf{k}_{\mathbb{V}}\right)$, there exists a largest open subset $U$ of $\mathbb{V}$ such that $\left.F\right|_{U}$ is locally constant, namely the union of all open subsets on which $F$ is locally constant. Moreover, $U$ is subanalytic in $\mathbb{V}$ since $U=\mathbb{V} \backslash \operatorname{Sing}(F)$. Note that $U \cap \operatorname{supp}(F)$ is again open in $\mathbb{V}$ and subanalytic. It is the largest open subset of $\mathbb{V}$ on which $F$ is locally constant with strictly positive rank. Hence it is a union of connected components of $U$.

Corollary 3.11. Let $F \in \operatorname{Mod}_{\mathbb{R c}, \gamma^{\circ a}}\left(\mathbf{k}_{\mathbb{V}}\right)$ and let $U=\operatorname{supp}(F) \backslash \operatorname{Sing}(F)$. Then $U$ is an open subset of $\mathbb{V}$ and $\left.F\right|_{U}$ is locally constant. Moreover, $U$ is dense in $\operatorname{supp}(F)$. In particular, one has $\operatorname{supp}(F)=\overline{\operatorname{Int}(\operatorname{supp}(F))}$.

Proof. We know already that $U$ is an open subset of $\mathbb{V}$ and $\left.F\right|_{U}$ is locally constant. It remains to prove that $U$ is dense in $\left\{x \in \mathbb{V} ; F_{x} \neq 0\right\}$. We may assume that $\gamma$ is polyhedral. Let $x \in X$ such that $F_{x} \nsucceq 0$. Applying Theorem 3.10, we find an open neighborhood $W$ of $x$ such that $F$ is constant on $\left(x+\gamma^{a}\right) \cap W$, hence on the open set $V:=\left(x+\operatorname{Int}\left(\gamma^{a}\right)\right) \cap W$. Then $x$ belongs to the closure of $V$ and $V \subset U$. 
Corollary 3.12. Let $F \in \operatorname{Mod}_{\mathbb{R c}, \gamma^{\circ a}}\left(\mathbf{k}_{\mathbb{V}}\right)$ and $G \in \operatorname{Mod}_{\mathbb{R c}, \gamma^{\circ}}\left(\mathbf{k}_{\mathbb{V}}\right)$. Then $\mathscr{E} x t^{j}(G, F) \simeq 0$ for $j \neq 0$.

Proof. Let $U$ and $V$ be the largest open subsets of $\mathbb{V}$ on which $F$ and $G$ are respectively locally constant. Then $W=U \cap V$ is open, dense and $\operatorname{R} \mathscr{H} o m(G, F)$ is concentrated in degree 0 on $W$. Therefore, $\operatorname{supp}\left(\mathscr{E} x t^{j}(G, F)\right)$ has empty interior for $j>0$. Since this sheaf belongs to $\operatorname{Mod}_{\mathbb{R c}, \gamma^{\circ a}}\left(\mathbf{k}_{\mathbb{V}}\right)$ by $[K S 90$, Prop. 5.4 .14$]$ and Corollary 1.7 , it must be 0 by Corollary 3.11 .

The result of Corollary 3.12 does not hold if both $F$ and $G$ belong to $\operatorname{Mod}_{\mathbb{R c}, \gamma^{\circ a}}\left(\mathbf{k}_{\mathbb{V}}\right)$.

Remark 3.13. One has R $\mathscr{H}$ om $\left(\mathbf{k}_{\gamma^{a}}, \mathbf{k}_{\operatorname{Int}(\gamma)}[n]\right) \simeq \mathbf{k}_{\{0\}}$. Indeed, Applying [KS90, Prop. 3.4.6]) we get

$$
\begin{aligned}
\operatorname{R} \mathscr{H} o m\left(\mathbf{k}_{\gamma^{a}}, \mathbf{k}_{\operatorname{Int}(\gamma)}[n]\right) & \simeq \operatorname{R} \mathscr{H o m}\left(\mathbf{k}_{\gamma^{a}}, \mathrm{D}^{\prime} \mathbf{k}_{\gamma}\right)[n] \\
& \simeq \operatorname{R} \mathscr{H} o m\left(\mathbf{k}_{\gamma^{a}} \otimes \mathbf{k}_{\gamma}, \mathbf{k}_{\mathbb{V}}\right)[n] \\
& \simeq \operatorname{R} \mathscr{H} o m\left(\mathbf{k}_{\{0\}}, \mathbf{k}_{\mathbb{V}}\right)[n] \simeq \mathbf{k}_{\{0\}} .
\end{aligned}
$$

Theorem 3.14. Assume (2.8). Let $\Omega$ be a $\gamma$-flat open set and let $Z=(\Omega+\gamma) \cap \overline{\Omega+\gamma^{a}}$, a $\gamma$-locally closed subset. Let $F \in \mathrm{D}_{\mathbb{R c}, \gamma^{\circ a}}^{\mathrm{b}}\left(\mathbf{k}_{\mathbb{V}}\right)$ and assume that $\left.F\right|_{\Omega}$ is locally constant. Then $\left.F\right|_{Z}$ is locally constant.

Proof. Let $x \in Z$ and let $U$ be an open convex neighborhood of $x$ such that $F(U) \stackrel{\sim}{\longrightarrow} F_{x}$ and such that, applying Theorem 3.10, $F$ is constant on $\left(x+\gamma^{a}\right) \cap U$. We choose a vector $v \in \operatorname{Int}\left(\gamma^{a}\right)$ and $\varepsilon>0$ such that $[x, x+\varepsilon v] \subset\left(x+\gamma^{a}\right) \cap U \subset Z$. Let $y=x+\varepsilon v$. Then $y \in \Omega$ and $F$ is constant on $[x, y]$. Set $W:=U \cap(y+\operatorname{Int}(\gamma))$ so that $x \in W$. We shall show that $F$ is constant on $W \cap Z$.

Let $z \in W \cap Z$. Then $[z, y] \subset \Omega+\gamma, y-z \in \gamma$ and $] z, y] \subset \overline{\left(\Omega+\gamma^{a}\right)}+\operatorname{Int}\left(\gamma^{a}\right)=\Omega+\gamma^{a}$. The last equality follows from Lemma 3.1. Hence we have $] z, y] \subset \Omega$ and $\left.F\right|_{[z, y]}$ is constant by Theorem 3.10. Consider the commutative diagram:

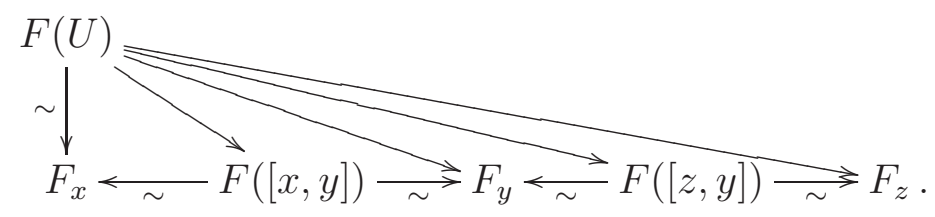

The horizontal arrows are isomorphism since $\left.F\right|_{[x, y]}$ and $\left.F\right|_{[z, y]}$ are constant. It follows that the map $F(U) \rightarrow F_{z}$ is an isomorphism.

\subsection{Piecewise linear $\gamma$-sheaves}

Definition 3.15. A PL- $\gamma$-stratification $(A, Z)$ of a closed set $S$ is a $\gamma$-barcode $(A, Z)$ such that $\operatorname{supp}(A, Z)=S$ and $Z_{\alpha} \cap Z_{\beta}=\varnothing$ for any $\alpha, \beta \in A$ with $\alpha \neq \beta$.

Lemma 3.16. Let $F \in \mathrm{D}_{\mathbb{R c}, \gamma^{\circ a}}^{\mathrm{b}}\left(\mathbf{k}_{\mathbb{V}}\right)$ and let $(A, Z)$ be a PL- $\gamma$-stratification of $\operatorname{supp}(F)$. Then $F_{x} \simeq 0$ for any $x \notin \bigcup_{\alpha \in A} Z_{\alpha}$. 
Proof. Assuming that $F_{x} \not 0$, let us show that $x \in \bigcup_{\alpha \in A} Z_{\alpha}$. By Theorem 3.10, there exists an open neighborhood $U$ of $x$ such that $\left.F\right|_{\left(x+\gamma^{a}\right) \cap U}$ is constant. Then one has $\left(x+\gamma^{a}\right) \cap U \subset \operatorname{supp}(F) \subset \bigcup_{\alpha \in A} \overline{Z_{\alpha}}$. Hence we obtain

$$
\begin{aligned}
x \in \overline{\left(x+\operatorname{Int}\left(\gamma^{a}\right)\right) \cap U} \subset \overline{\left(x+\operatorname{Int}\left(\gamma^{a}\right)\right) \cap\left(\bigcup_{\alpha \in A} \overline{Z_{\alpha}}\right)} & =\bigcup_{\alpha \in A} \overline{\left(x+\operatorname{Int}\left(\gamma^{a}\right)\right) \cap \overline{Z_{\alpha}}} \\
& =\bigcup_{\alpha \in A} \overline{\left(x+\operatorname{Int}\left(\gamma^{a}\right)\right) \cap Z_{\alpha}} .
\end{aligned}
$$

Hence there exists $\alpha \in A$ such that $x \in \overline{\left(x+\operatorname{Int}\left(\gamma^{a}\right)\right) \cap Z_{\alpha}}$. Then Lemma 3.3 (b) implies that $x \in Z_{\alpha}$.

Recall that for $F \in \mathrm{D}_{\mathbb{R} c, \gamma^{\circ a}}^{\mathrm{b}}\left(\mathbf{k}_{\mathbb{V}}\right), \operatorname{Sing}(F)$ denotes its singular locus.

Theorem 3.17. Assume (2.8). Let $F \in \mathrm{D}_{\mathrm{PL}, \gamma^{\circ a}}^{\mathrm{b}}\left(\mathbf{k}_{\mathbb{V}}\right)$. Then there exists a PL- $\gamma$ stratification $(A, Z)$ of $\operatorname{supp}(F)$ such that $\left.F\right|_{Z_{\alpha}}$ is constant for each $\alpha \in A$.

Proof. (i) Assume first that $\operatorname{supp}(F)$ is compact.

(a) Since $F$ is PL, there exists a finite family of affine hyperplanes $\left\{H_{a}\right\}_{a \in A}$ such that $\operatorname{Sing}(F) \subset \bigcup_{a \in A} H_{a}$. On the other hand, $\operatorname{Sing}(F)$ has pure codimension 1, thanks to Proposition 3.7. Let $B=\left\{a \in A ; \operatorname{Int}_{H_{a}}\left(\operatorname{Sing}(F) \cap H_{a}\right) \neq \varnothing\right\}$. Then, $\operatorname{Sing}(F) \cap\left(\bigcup_{a \in B} H_{a}\right)$ is dense in $\operatorname{Sing}(F)$. Hence we obtain $\operatorname{Sing}(F) \subset \bigcup_{a \in B} H_{a}$. Set $\Omega=\operatorname{supp}(F) \backslash\left(\bigcup_{a \in B} H_{a}\right)$. Then $\Omega$ is an open subset of $\mathbb{V}$ and dense in $\operatorname{supp}(F)$ by Corollary 3.11.

(b) Let $\Omega=\bigsqcup_{i \in I} \Omega_{i}$ be the decomposition of $\Omega$ into connected components. Then each $\Omega_{i}$ is an open convex polytope.

At generic points of $H_{a}(a \in B)$, one has $\dot{T}_{H_{a}}^{*} \mathbb{V} \cap \mu \operatorname{supp}(F) \neq \varnothing$ by Proposition 3.7. If $H_{a}=\{\langle x, \xi\rangle=c\}$, one has $\pm \xi \in \gamma^{\circ a}$ and thus $H_{a} \cap(x+\operatorname{Int}(\gamma))=\varnothing$ for any $x \in H_{a}$. Denote by $H_{a}^{ \pm}$the two open half-spaces with boundary $H_{a}$. These are $\gamma$-flat open sets and it follows that any connected component $\Omega_{i}$ of $\Omega$, which is a finite intersection of such half-spaces, is also $\gamma$-flat.

Set $Z_{i}=\left(\Omega_{i}+\gamma\right) \cap \overline{\Omega_{i}+\gamma^{a}}$. Then each $Z_{i}$ is $\gamma$-locally closed and $\operatorname{supp}(F)=\bigcup_{i \in I} \overline{Z_{i}}$. By Lemma 3.5, $Z_{i} \cap Z_{j}=\varnothing$ if $i \neq j$. Hence $\left\{Z_{i}\right\}_{i \in I}$ is a $\gamma$-stratification of $\operatorname{supp}(F)$. By Proposition $3.14,\left.F\right|_{Z_{i}}$ is locally constant. Since $Z_{i}$ is convex, $\left.F\right|_{Z_{i}}$ is constant.

(ii) Now we consider the general case where $\operatorname{supp}(F)$ is not necessarily compact.

Taking $v \in \operatorname{Int}(\gamma)$, one sets $U_{n}=-n v+\operatorname{Int}(\gamma)$ and $S_{n}=n v+\gamma^{a}$ for $n \in \mathbb{Z}$. Then $\left\{U_{n}\right\}_{n \in \mathbb{Z}}$ is an increasing family of $\gamma$-open subsets, and $\left\{S_{n}\right\}_{n \in \mathbb{Z}}$ is an increasing family of $\gamma$-closed subsets. Moreover, one has $\mathbb{V}=\bigcup_{n \in \mathbb{Z}} U_{n}=\bigcup_{n \in \mathbb{Z}} S_{n}$ and $\bigcap_{n \in \mathbb{Z}} U_{n}=$ $\bigcap_{n \in \mathbb{Z}} S_{n}=\varnothing$. Set $I=\mathbb{Z} \times \mathbb{Z}$ and, for $i=(m, n) \in I$, set $K_{i}:=\left(U_{m} \backslash U_{m-1}\right) \cap\left(S_{n} \backslash S_{n-1}\right)$. Then $\left\{K_{i}\right\}_{i \in I}$ is a locally finite family of $\gamma$-locally closed subsets such that $\mathbb{V}=\bigsqcup_{i \in I} K_{i}$.

Set $F_{i}=F \otimes \mathbf{k}_{K_{i}}$. Then $F_{i} \in \mathrm{D}_{\mathrm{PL}, \gamma^{\circ a}}^{\mathrm{b}}\left(\mathbf{k}_{\mathbb{V}}\right)$ and $\operatorname{supp}\left(F_{i}\right)$ is compact. Hence by Step (i) there exists a finite PL- $\gamma$-stratification $\left\{Z_{\alpha}\right\}_{\alpha \in A_{i}}$ of $\operatorname{supp}\left(F_{i}\right)$ such that $\left.F_{i}\right|_{Z_{\alpha}}$ is constant for each $\alpha \in A_{i}$. Then, setting $A=\bigsqcup A_{i}$, we obtain a desired PL- $\gamma$ stratification $\left\{Z_{\alpha}\right\}_{\alpha \in A}$ of $\operatorname{supp}(F)$. Note that $Z_{\alpha} \cap Z_{\alpha^{\prime}}=\varnothing$ for $\alpha \neq \alpha^{\prime}$ follows again from Lemma 3.5. Indeed, assume that $\alpha \in A_{i}$ and $\alpha^{\prime} \in A_{i^{\prime}}$. If $i=i^{\prime}$, one has obviously 
$Z_{\alpha} \cap Z_{\alpha^{\prime}}=\varnothing$. If $i \neq i^{\prime}$, then $\operatorname{Int}\left(Z_{\alpha}\right) \cap \operatorname{Int}\left(Z_{\alpha^{\prime}}\right) \subset K_{i} \cap K_{i^{\prime}}=\varnothing$, and Lemma 3.5 implies that $Z_{\alpha} \cap Z_{\alpha^{\prime}}=\varnothing$.

Remark 3.18. In the course of the proof of Theorem 3.17, we have also obtained the following result.

Let $F \in \mathrm{D}_{\mathbb{R} \text {, } \gamma^{\circ a}}^{\mathrm{b}}\left(\mathbf{k}_{\mathbb{V}}\right)$, and let $\left\{H_{a}\right\}_{a \in A}$ be a locally finite family of affine hyperplanes such that $\operatorname{Sing}(F) \subset \bigcup_{a \in A} H_{a}$. Then, one has $\operatorname{Sing}(F) \subset \bigcup_{a \in B} H_{a}$ where $B=\left\{a \in A ; \operatorname{Int}_{H_{a}}\left(\operatorname{Sing}(F) \cap H_{a}\right) \neq \varnothing\right\}$. Moreover, $F$ is PL by Lemma 3.16.

As usual, for an additive category $\mathscr{C}$, we shall denote by $\mathrm{C}^{\mathrm{b}}(\mathscr{C})$ the category of bounded complexes of objects of $\mathscr{C}$.

Conjecture 3.19. Let $F \in \mathrm{D}_{\mathrm{PL}, \gamma^{\circ \mathrm{a}}}^{\mathrm{b}}\left(\mathbf{k}_{\mathbb{V}}\right)$ and assume that $F$ has compact support. Then there exists a bounded complex $F^{\bullet} \in \mathrm{C}^{\mathrm{b}}\left(\operatorname{Mod}_{\mathrm{PL}, \gamma^{\circ a}}\left(\mathbf{k}_{\mathbb{V}_{\gamma}}\right)\right)$ whose image in $\mathrm{D}_{\mathrm{PL}, \gamma^{\circ a}}^{\mathrm{b}}\left(\mathbf{k}_{\mathbb{V}}\right)$ is isomorphic to $F$ and such that each component $F^{j}$ of $F^{\bullet}$ is a barcode $\gamma$-sheaf (see Definition 2.16) with compact support.

\section{References}

[BL16] Ulrich Bauer and Michael Lesnick, Persistent diagram as diagrams: a categorification of the stability theorem (2016), available at arXiv:1610.10085.

[BdSS15] Peter Bubenik, Vin de Silva, and Jonathan Scott, Metrics for generalized persistence modules, Found. Comput.math. 15 (2015), 1501-1531, available at arXiv:1312.3829.

[BS14] Peter Bubenik and Jonathan Scott, Categorification of persistent homology, Discrete Comput. Geom. 51 (2014), 600-627, available at arXiv: 1205.3669.

[CCSG ${ }^{+}$09] Frédéric Chazal, David Cohen-Steiner, M Glisse, L. J. Guibas, and Steve Oudot, Proximity of Persistence Modules and their Diagrams, Proc. 25th, ACM Sympos. on Comput. Geom., 2009, pp. 237-246.

[CdSGO16] Frédéric Chazal, Vin de Silva, M Glisse, and Steve Oudot, The Structure and Stability of Persistence Modules (Springer, ed.), Springer Briefs in Mathematics, 2016.

[CSEH07] David Cohen-Steiner, Herbert Edelsbrunner, and John Harer, Stability of persistence diagrams, Discrete Comput. Geom. 37 (2007), 103-120.

[CB14] William Crawley-Boevey, Decomposition of pointwise finite-dimensional persistence modules, J. Algebra Appl. 14 (2014), 1550066, 8 pp, available at arXiv:1210.0819.

[Cur13] Justin M. Curry, Sheaves, cosheaves and applications (2013), available at arXiv: $1303.3255 \mathrm{v} 2$.

[EH08] Herbert Edelsbrunner and John Harer, Persistent homology - a survey, Surveys on Discrete and Computational Geometry. Twenty Years Later, 2008, pp. 257-282.

[Ghr08] Robert Ghrist, Barcodes: The persistent topology of data, Bull. Amer. Math. Soc. 45 (2008), 61-75.

[GRS83] L Guibas, L Ramschaw, and J Stolfi, A kinetic framework for computational geometry, Proc. IEEE Symp. on Foundations of Computer Science (1983), 74-123.

[Gui16] Stéphane Guillermou, The three cusps conjecture (2016), available at arXiv:1603.07876.

[GKS12] Stéphane Guillermou, Masaki Kashiwara, and Pierre Schapira, Sheaf quantization of Hamiltonian isotopies and applications to nondisplaceability problems, Duke Math Journal 161 (2012), 201-245. 
[GS14] Stéphane Guillermou and Pierre Schapira, Microlocal theory of sheaves and Tamarkin's non displaceability theorem, LN of the UMI (2014), 43-85, available at arXiv:1106.1576.

[GM88] Mark Goresky and Robert MacPherson, Stratified Morse Theory, Ergebnisse Der Mathematik Und Ihrer Grenzgebiete, vol. 14, Springer-Verlag, Berlin, 1988.

[Kas75] Masaki Kashiwara, On the maximally overdetermined systems of linear differential equations I, Publ. Res. Inst. Math. Sci. 10 (1975), 563-579.

[Kas84] _ The Riemann-Hilbert problem for holonomic systems, Publ. RIMS, Kyoto Univ. 20 (1984), 319-365.

[KS90] Masaki Kashiwara and Pierre Schapira, Sheaves on manifolds, Grundlehren der Mathematischen Wissenschaften [Fundamental Principles of Mathematical Sciences], vol. 292, Springer-Verlag, Berlin, 1990.

[Les15] Michael Lesnick, The Theory of the Interleaving Distance on Multidimensional Persistence Modules, Found. Comput. Math. 15 (2015), 613-650.

[LW15] Michael Lesnick and Matthew Wright, Interactive Visualization of 2-D Persistence Modules (2015), available at arXiv: 1512.00180.

[Oud15] Steve Oudot, Persistence Theory: From Quiver Representations to Data Analysis, Mathematical Surveys and Monographs, vol. 209, AMS, 2015.

[Sch91] Pierre Schapira, Operations on constructible functions, Journal of Pure and Applied Algebra 72 (1991), 83-93.

[Tam08] Dmitry Tamarkin, Microlocal conditions for non-displaceability (2008), available at arXiv:0809.1584.

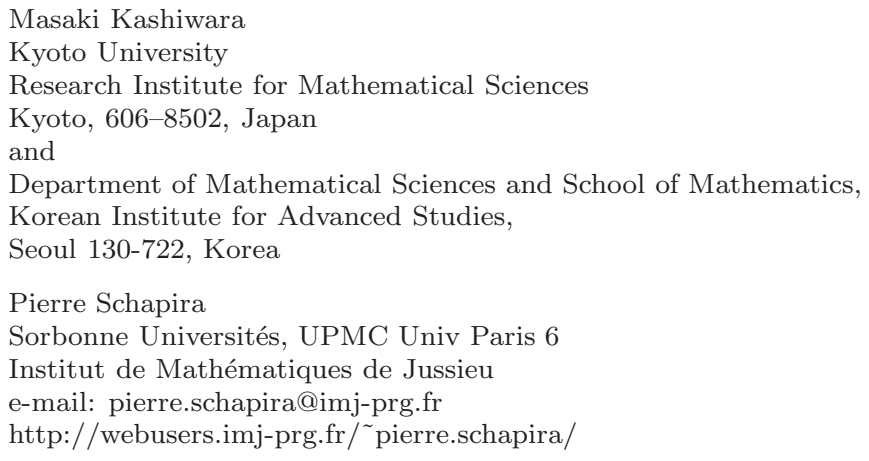

